\title{
SPECTRA AND CATENARITY OF MULTI-PARAMETER QUANTUM SCHUBERT CELLS*
}

\author{
MILEN YAKIMOV \\ Department of Mathematics, Louisiana State University, Baton Rouge, LA 70803 USA \\ email:yakimov@math.lsu.edu
}

\begin{abstract}
We study the ring theory of the multi-parameter deformations of the quantum Schubert cell algebras obtained from 2-cocycle twists. This is a large family, which extends the Artin-Schelter-Tate algebras of twisted quantum matrices. We classify set theoretically the spectra of all such multi-parameter quantum Schubert cell algebras, construct each of their prime ideals by contracting from explicit normal localizations and prove formulas for the dimensions of their Goodearl-Letzter strata for base fields of arbitrary characteristic and all deformation parameters that are not roots of unity. Furthermore, we prove that the spectra of these algebras are normally separated and that all such algebras are catenary.
\end{abstract}

2010 Mathematics Subject Classification. Primary 16W35; Secondary 20G42, $14 \mathrm{M} 15$.

1. Introduction. The quantum Schubert cell algebras (or the quantum nilpotent algebras) form a large family of iterated Ore extensions defined by De Concini et al. [9] and Lusztig [30]. These are subalgebras of the negative part of a quantized universal enveloping algebra $\mathcal{U}_{q}(\mathfrak{g})$ defined over an arbitrary base field $\mathbb{K}$ and are indexed by the elements of the Weyl group $W$ of $\mathfrak{g}$. The algebra corresponding to $w \in W$, to be denoted by $\mathcal{U}_{-}^{w}$, is a deformation of the universal enveloping algebra of the nilpotent Lie algebra $\mathfrak{n}_{-} \cap w\left(\mathfrak{n}_{+}\right)$, where $\mathfrak{n}_{ \pm}$are the niradicals of a pair of opposite Borel subalgebras of $\mathfrak{g}$. Furthermore, the algebras $\mathcal{U}_{-}^{w}$ are isomorphic [37] to certain localization of quotients of homogeneous coordinate rings of quantum partial flag varieties, similar to the classical case of Schubert cells. They specialize [39] to the coordinate rings of Schubert cells equipped with the standard Poisson structure $[5,19]$.

There has been a great interest in the ring theoretic study of these algebras in an attempt to develop a somewhat general theory for iterated Ore extensions. The algebra $\mathcal{U}_{-}^{w}$ admits a natural rational action of the torus $\mathbb{T}^{|\mathcal{S}(w)|}=\left(\mathbb{K}^{*}\right)^{\times|\mathcal{S}(w)|}$ by algebra automorphisms, where $\mathcal{S}(w)$ is the support of $w$, see Section 2.4. The $\mathbb{T}^{|\mathcal{S}(w)|}$-prime spectrum of $\mathcal{U}_{-}^{w}$ was described in $[33,38]$, and the dimensions of the corresponding Goodearl-Letzter strata were computed in $[3,39]$. The Goodearl-Lenagan conjecture [16] on polynormality of the torus invariant prime ideals of quantum matrices and more generally the algebras $\mathcal{U}_{-}^{w}$ were proved in [40], where catenarity and normal separation of the spectra of $\mathcal{U}_{-}^{w}$ was established too. The algebras $\mathcal{U}_{-}^{w}$ play an important role in various other contexts: Heckenberger-Schneider classified [22] the homogeneous coideal subalgebras of quantized universal enveloping algebras of Borel subalgebras

* Dedicated to Kenny Brown and Toby Stafford on the occasion of their 60th birthdays 
in terms of $\mathcal{U}_{-}^{w}$, and Geiß-Leclerc-Schröer proved [12] that $\mathcal{U}_{-}^{w}$ are quantum cluster algebras for simply laced $\mathfrak{g}$, char $\mathbb{K}=0$, and $q \in \mathbb{K}$ transcendental over $\mathbb{Q}$.

Artin et al. proved [1] that multi-parameter versions of the algebras of quantum matrices and the quantum linear groups can be obtained from the single parameter case by 2-cocycle twists. After this a number of authors investigated the effect of such twists on the spectra of graded algebras. The spectra of multi-parameter quantum groups were studied by Hodges-Levasseur-Toro [21] and Costantini-Varagnolo [10]. The spectra of special cases of multi-parameter quantum Schubert cell algebras were investigated by many authors: $[\mathbf{1 5}, \mathbf{2 3}, \mathbf{3 5}, \mathbf{3 6}$, to name a few. Nowlin and Johnson $[25,34]$ proved that certain interesting classes of algebras defined in a completely independent way turn out to be isomorphic to special twists of quantum Schubert cell algebras for affine Kac-Moody algebras.

In this paper we carry out a general study of the ring theory of twisted quantum Schubert cell algebras when $q$ is not a root of unity (without any assumptions on the base field $\mathbb{K})$. The algebra $\mathcal{U}_{-}^{w}$ is graded by the subgroup $\mathcal{Q}_{\mathcal{S}(w)}$ of the root lattice of $\mathfrak{g}$, generated by the simple roots in the support $\mathcal{S}(w)$ of $w \in W$, see Section 3. For $\mathbf{p} \in Z^{2}\left(\mathcal{Q}_{\mathcal{S}(w)}, \mathbb{K}^{*}\right)$, denote by $\mathcal{U}_{-, \mathbf{p}}^{w}$ the algebra obtained by a cocycle twist from $\mathcal{U}_{-}^{w}$ using p. Firstly, we give an explicit classification of $\operatorname{Spec}_{\mathcal{U}_{-, \mathbf{p}}^{w}}^{w}$. This is stated in Theorem 3.1. As in [37, 40], we use results of Joseph [26, 27] and Gorelik [20]. Most of those results were formulated in $[\mathbf{2 7}, \mathbf{2 0}]$ for $\mathbb{K}=k(q)$, char $k=0$. We show that the proofs of all such needed results work for an arbitrary $q \in \mathbb{K}^{*}$, which is not a root of unity and without restrictions on the characteristic of $\mathbb{K}$. At some steps we take shortcuts using results of Goodearl and Letzter [18] and [4, Section II.6]. Furthermore, Theorem 3.1 expresses each prime ideal of $\mathcal{U}_{-, \mathbf{p}}^{w}$ as a contraction from an explicit normal localization of a quotient of $\mathcal{U}_{-, \mathbf{p}}^{w}$ by a $\mathbb{T}^{|\mathcal{S}(w)|}$-prime. These localizations are smaller than the ones obtained via the Cauchon method of deleting derivations [7, 33].

The spectra $\operatorname{Spec} \mathcal{U}_{-, \mathbf{p}}^{w}$ are partitioned [18] into a union of Goodearl-Letzter strata, which are isomorphic to the spectra of Laurent polynomial rings. We prove an explicit formula for the dimensions of the latter in Theorem 4.5, which works for all 2-cocycles $\mathbf{p}, q \in \mathbb{K}^{*}$ not a root of unity, and arbitrary base fields $\mathbb{K}$. In the special one-parameter case this formula was obtained by Bell et al. [3] and Yakimov [39] when char $\mathbb{K}=0$ and $q$ is transcendental over $\mathbb{Q}$. We give a (very short!) new proof of the one-parameter case in Proposition 4.1.

Furthermore, using results of [40] we prove in an explicit way that the $\mathbb{T}^{|\mathcal{S}(w)|}$ invariant prime ideals of the algebras $\mathcal{U}_{-, \mathbf{p}}^{w}$ are equivariantly polynormal. In the special case of multi-parameter quantum matrices this gives a constructive proof of a conjecture of Brown and Goodearl [4, Conjecture II.10.9]. Moreover, we show that $\operatorname{Spec} \mathcal{U}_{-, \mathbf{p}}^{w}$ are normally separated and that all algebras $\mathcal{U}_{-, \mathbf{p}}^{w}$ are catenary. This provides a very large class of iterated Ore extensions for which Gabber's theorem on catenarity of universal enveloping algebras of solvable Lie algebras can be extended. In a related direction, in a forthcoming publication we will prove another conjecture of Brown and Goodearl [4, Conjecture II.10.7] that all prime ideals of multi-parameter quantum groups are completely prime under mild conditions of the cocycle twist.

\section{Quantum groups, quantum Schubert cells and their twists.}

2.1. Quantized universal enveloping algebras. Denote $\mathbb{N}=\{0,1, \ldots\}$ and $\mathbb{Z}_{+}=$ $\{1,2, \ldots\}$. For $m \leq n \in \mathbb{Z}$ set $[m, n]=\{m, \ldots, n\}$. Throughout the paper, $\mathbb{K}$ will denote 
a base field (of arbitrary characteristic), and $q \in \mathbb{K}^{*}$ will denote an element which is not a root of unity. We fix a simple Lie algebra $\mathfrak{g}$ of rank $r$ with Cartan matrix $\left(c_{i j}\right)$. Let $\mathcal{U}_{q}(\mathfrak{g})$ be the quantized universal enveloping algebra of $\mathfrak{g}$ over $\mathbb{K}$ with deformation parameter $q$. Recall [24] that $\mathcal{U}_{q}(\mathfrak{g})$ is the $\mathbb{K}$-algebra with generators

$$
X_{i}^{ \pm}, K_{i}^{ \pm 1}, \quad i \in[1, r]
$$

and relations

$$
\begin{gathered}
K_{i}^{-1} K_{i}=K_{i} K_{i}^{-1}=1, K_{i} K_{j}=K_{j} K_{i}, K_{i} X_{j}^{ \pm} K_{i}^{-1}=q_{i}^{ \pm c_{i j}} X_{j}^{ \pm}, \\
X_{i}^{+} X_{j}^{-}-X_{j}^{-} X_{i}^{+}=\delta_{i, j} \frac{K_{i}-K_{i}^{-1}}{q_{i}-q_{i}^{-1}}, \sum_{k=0}^{1-c_{i j}}(-1)^{k}\left[\begin{array}{c}
1-c_{i j} \\
k
\end{array}\right]_{q_{i}}\left(X_{i}^{ \pm}\right)^{k} X_{j}^{ \pm}\left(X_{i}^{ \pm}\right)^{1-c_{i j}-k}=0, i \neq j,
\end{gathered}
$$

where $q_{i}=q^{d_{i}}$ and $\left\{d_{i}\right\}_{i=1}^{r}$ is the collection of positive relatively prime integers such that $\left(d_{i} c_{i j}\right)$ is symmetric. Moreover, $\mathcal{U}_{q}(\mathfrak{g})$ is a Hopf algebra with co-multiplication, antipode and co-unit given by

$$
\Delta\left(K_{i}\right)=K_{i} \otimes K_{i}, \Delta\left(X_{i}^{+}\right)=X_{i}^{+} \otimes 1+K_{i} \otimes X_{i}^{+}, \Delta\left(X_{i}^{-}\right)=X_{i}^{-} \otimes K_{i}^{-1}+1 \otimes X_{i}^{-}
$$

and

$$
S\left(K_{i}\right)=K_{i}^{-1}, S\left(X_{i}^{+}\right)=-K_{i}^{-1} X_{i}^{+}, S\left(X_{i}^{-}\right)=-X_{i}^{-} K_{i}, \epsilon\left(K_{i}\right)=1, \epsilon\left(X_{i}^{ \pm}\right)=0 .
$$

The subalgebras of $\mathcal{U}_{q}(\mathfrak{g})$ generated by $\left\{X_{1}^{ \pm}, \ldots, X_{r}^{ \pm}\right\}$will be denoted by $\mathcal{U}_{ \pm}$. The sets of simple roots, simple co-roots and fundamental weights of $\mathfrak{g}$ will be denoted by $\left\{\alpha_{i}\right\}_{i=1}^{r}$, $\left\{\alpha_{i}^{\vee}\right\}_{i=1}^{r}$ and $\left\{\omega_{i}\right\}_{i=1}^{r}$. The root and weight lattices of $\mathfrak{g}$ will be denoted by $\mathcal{Q}$ and $\mathcal{P}$. Let $\mathcal{Q}^{+}=\mathbb{N} \alpha_{1}+\ldots+\mathbb{N} \alpha_{r}, \mathcal{P}^{+}=\mathbb{N} \omega_{1}+\ldots+\mathbb{N} \omega_{r}$, and $\mathcal{P}^{++}=\mathbb{Z}_{+} \omega_{1}+\ldots+\mathbb{Z}_{+} \omega_{r}$. Recall the standard partial order on $\mathcal{P}$ : for $v_{1}, v_{2} \in \mathcal{P}$ set $v_{1} \geq v_{2}$ if $v_{2}=v_{1}-\gamma$ for some $\gamma \in \mathcal{Q}^{+}$. Let $v_{1}>v_{2}$ if $v_{1} \geq v_{2}$ and $v_{1} \neq v_{2}$. If $\lambda=\sum_{i \in I} n_{i} \omega_{i} \in \mathcal{P}^{+}, n_{i}>0, \forall i \in I$ we will say that the support of $\lambda$ is $I$. Denote by $\langle.,$.$\rangle the symmetric bilinear form$ on $\operatorname{Span}_{\mathbb{Q}}\left\{\alpha_{i}\right\}_{i=1}^{r}$ such that $\left\langle\alpha_{i}, \alpha_{j}\right\rangle=d_{i} c_{i j}, \forall i, j \in[1, r]$. The $q$-weight spaces of a $\mathcal{U}_{q}(\mathfrak{g})$ module $V$ are defined by

$$
V_{\nu}=\left\{v \in V \mid K_{i} v=q^{\left\langle v, \alpha_{i}\right\rangle} v, \forall i \in[1, r]\right\}, v \in \mathcal{P} .
$$

A type one $\mathcal{U}_{q}(\mathfrak{g})$-module is a $\mathcal{U}_{q}(\mathfrak{g})$-module such that $V=\oplus_{\nu \in \mathcal{P}} V_{\nu}$. The category of (left) finite dimensional type one $\mathcal{U}_{q}(\mathfrak{g})$-modules is semi-simple (see [24, Theorem 5.17 and Remark on p. 85] for the validity of this for general base fields $\mathbb{K}$ and $q \in \mathbb{K}^{*}$ not a root of unity). Furthermore, this category is closed under taking tensor products and duals, where the latter are defined as left $\mathcal{U}_{q}(\mathfrak{g})$-modules using the antipode of $\mathcal{U}_{q}(\mathfrak{g})$. The irreducible modules in this category are parametrized [24, Theorem 5.10] by the set of dominant integral weights $\mathcal{P}^{+}$. Let $V(\lambda)$ denote the irreducible type one $\mathcal{U}_{q}(\mathfrak{g})$-module of the highest weight $\lambda \in \mathcal{P}^{+}$.

Let $W$ and $\mathcal{B}_{\mathfrak{g}}$ be the Weyl and braid groups of $\mathfrak{g}$, and let $s_{1}, \ldots, s_{r}$ and $T_{1}, \ldots, T_{r}$ be their standard generating sets corresponding to the simple roots $\alpha_{1}, \ldots, \alpha_{r}$. Denote by $\ell: W \rightarrow \mathbb{N}$ the standard length function. The braid group $\mathcal{B}_{\mathfrak{g}}$ acts on $\mathcal{U}_{q}(\mathfrak{g})$ by algebra automorphisms by [24, equations 8.14(2), (3), (7) and (8)] and on the finite dimensional type one $\mathcal{U}_{q}(\mathfrak{g})$-modules by [24, equation $\left.8.6(2)\right]$. These actions are compatible: $T_{w}(x . v)=\left(T_{w} x\right) .\left(T_{w} v\right)$ for all $w \in W, x \in \mathcal{U}_{q}(\mathfrak{g}), v \in V(\lambda), \lambda \in \mathcal{P}^{+}$. 
2.2. Quantum function algebras. If $\mathbb{K}$ is an algebraically closed field of characteristic 0 , we will denote by $G$ the connected simply connected algebraic group with the Lie algebra $\mathfrak{g}$. For all base fields $\mathbb{K}$ and deformation parameters $q \in \mathbb{K}^{*}$ which are not roots of unity, one defines the quantum group $R_{q}[G]$ as the Hopf subalgebra of the restricted dual $\left(\mathcal{U}_{q}(\mathfrak{g})\right)^{\circ}$ equal to the span of the matrix coefficients of the modules $V(\lambda), \lambda \in \mathcal{P}^{+}$:

$$
c_{\xi, v}^{\lambda} \in\left(\mathcal{U}_{q}(\mathfrak{g})\right)^{\circ}, \quad c_{\xi, v}^{\lambda}(x)=\xi(x v), \quad v \in V(\lambda), \xi \in V(\lambda)^{*}, x \in \mathcal{U}_{q}(\mathfrak{g}) .
$$

Since we work with arbitrary base fields, $G$ is merely a symbol. The canonical left and right actions of $\mathcal{U}_{q}(\mathfrak{g})$ on $\mathcal{U}_{q}(\mathfrak{g})^{\circ}$

$$
x \rightarrow c=\sum c_{(2)}(x) c_{(1)}, c<x=\sum c_{(1)}(x) c_{(2)}, x \in \mathcal{U}_{q}(\mathfrak{g}), c \in R_{q}[G]
$$

preserve $R_{q}[G]$, where $\Delta(c)=\sum c_{(1)} \otimes c_{(2)}$.

For each $\lambda \in \mathcal{P}^{+}$we fix the highest weight vector $v_{\lambda}$ of $V(\lambda)$ and denote

$$
c_{\xi}^{\lambda}=c_{\xi, v_{\lambda}}^{\lambda} \in R_{q}[G] .
$$

The subspace

$$
R^{+}=\operatorname{Span}\left\{c_{\xi}^{\lambda} \mid \lambda \in \mathcal{P}^{+}, \xi \in V(\lambda)^{*}\right\} \subset R_{q}[G]
$$

is a subalgebra of $R_{q}[G]$. We will need the $R$-matrix commutation relations in $R^{+}$. Denote the canonical $\mathcal{Q}$-grading of $\mathcal{U}_{q}(\mathfrak{g})$ :

$$
\text { wt } X_{i}^{ \pm}= \pm \alpha_{i} \text {, wt } K_{i}=0, \quad i \in[1, r] \text {. }
$$

For $\gamma \in \mathcal{Q}^{+}, \gamma \neq 0$ set $m(\gamma)=\operatorname{dim}\left(\mathcal{U}_{+}\right)_{\gamma}=\operatorname{dim}\left(\mathcal{U}_{-}\right)_{-\gamma}$. Denote by $\left\{u_{\gamma, i}\right\}_{i=1}^{m(\gamma)}$ and $\left\{u_{-\gamma, i}\right\}_{i=1}^{m(\gamma)}$ a pair of dual bases of $\left(\mathcal{U}_{+}\right)_{\gamma}$ and $\left(\mathcal{U}_{-}\right)_{-\gamma}$ with respect to the Rosso-Tanisaki form (see [24, Chap. 6] for a discussion of the properties of this form for arbitrary base fields $\mathbb{K})$. Then we have:

$$
c_{\xi_{1}}^{\lambda_{1}} c_{\xi_{2}}^{\lambda_{2}}=q^{\left\langle\lambda_{1}, \lambda_{2}\right\rangle-\left\langle\nu_{1}, \nu_{2}\right\rangle} c_{\xi_{2}}^{\lambda_{2}} c_{\xi_{1}}^{\lambda_{1}}+\sum_{\gamma \in \mathcal{Q}^{+}, \gamma \neq 0} \sum_{i=1}^{m(\gamma)} q^{\left\langle\lambda_{1}, \lambda_{2}\right\rangle-\left\langle\nu_{1}-\gamma, \nu_{2}+\gamma\right\rangle} c_{S^{-1}\left(u_{\gamma, i}\right) \xi_{2}}^{\lambda_{2}} c_{S^{-1}\left(u_{-\gamma, i}\right) \xi_{1}}^{\lambda_{1}},
$$

for all $\lambda_{i} \in \mathcal{P}^{+}, v_{i} \in \mathcal{P}$ and $\xi_{i} \in\left(V\left(\lambda_{i}\right)^{*}\right)_{v_{i}}$, see e.g. [4, Theorem I.8.15].

For $\lambda \in \mathcal{P}^{+}$and $w \in W$, let $\xi_{w, \lambda} \in\left(V(\lambda)^{*}\right)_{-w \lambda}$ such that $\left\langle\xi_{w, \lambda}, T_{w} v_{\lambda}\right\rangle=1$. (Since $T_{w}\left(V(\lambda)_{\lambda}\right)=V(\lambda)_{w \lambda}, \operatorname{dim} V(\lambda)_{w \lambda}=1$.) Define

$$
e_{w}^{\lambda}=c_{\xi_{w, \lambda}}^{\lambda}, \quad \lambda \in \mathcal{P}^{+}, w \in W .
$$

Then

$$
e_{w}^{\lambda_{1}} e_{w}^{\lambda_{2}}=e_{w}^{\lambda_{1}+\lambda_{2}}=e_{w}^{\lambda_{2}} e_{w}^{\lambda_{1}}, \quad \forall \lambda_{1}, \lambda_{2} \in \mathcal{P}^{+}, w \in W,
$$

see [40, equation (2.18)]. Denote the multiplicative subsets $E_{w}=\left\{e_{w}^{\lambda} \mid \lambda \in \mathcal{P}^{+}\right\} \subset R^{+}$.

LeMma 2.1. ([27, Lemma 9.1.10]). For all $w \in W, E_{w}$ is an Ore subset in $R^{+}$.

In [27] this result was stated for fields $\mathbb{K}$ of characteristic 0 (see [27, Section A.2.9]), but Joseph's proof [27] works for all base fields $\mathbb{K}, q \in \mathbb{K}^{*}$ not a root of unity as we see 
below. By (2.4) the set $E_{w_{0}}$ consists of normal elements of $R^{+}$. Therefore, $\left\{e^{n} \mid n \in \mathbb{N}\right\}$ is an Ore subset of $R^{+}$for all $e \in E_{w_{0}}$, in particular $E_{w_{0}}$ is an Ore subset of $R^{+}$. Joseph proved iteratively that

$$
\left\{e^{n} \mid n \in \mathbb{N}\right\} \text { are Ore subsets of } R^{+} \text {for all } e \in E_{w}, w \in W
$$

using the following procedure. Let $\sigma$ be an automorphism of a ring $A$, and $\partial$ be a locally nilpotent (right skew) $\sigma$-derivation of $A$ (i.e. for all $a, b \in A, \partial(a b)=\partial(a) \sigma(b)+$ $a \partial(b))$ such that $\sigma \partial \sigma^{-1}$ is a scalar multiple of $\partial$. The degree $\operatorname{deg}_{\partial} a$ of an element $a \in A \backslash\{0\}$ is defined as the minimal positive integer $m$ such that $\partial^{m+1}(a)=0$. Such a skew derivation $\partial$ is called right regular if for all $\sigma$-eigenvectors $a, b \in A$ of $\partial$-degree $m$ and $n$, respectively, and $k \in[n, m+n]$

$$
\partial^{k}(a b)=\sum_{i=0}^{m+n-k} s_{i}\left(\partial^{k-n+i} a\right)\left(\partial^{n-i} b\right)
$$

for some $s_{0}, \ldots, s_{m+n-k} \in \mathbb{K}, s_{0} \neq 0$. It is straightforward to check that if $\sigma \partial \sigma^{-1}=q^{\prime} \partial$ for some $q^{\prime} \in \mathbb{K}^{*}$, which is not a root of unity, then $\partial$ is right regular, since in that case all coefficients $s$ are products of $q^{\prime}$-binomial coefficients and $\sigma$-eigenvalues. Joseph's iterative proof of Lemma 2.1 relies on the following fact [27, Lemma A.2.9]:

If $\left\{e^{n} \mid n \in \mathbb{N}\right\}$ is an Ore subset of $A$ and $e \in A$ is a $\sigma$-eigenvector of degree $\operatorname{deg}_{\partial} e=m$, then $\left\{\left(\partial^{m} e\right)^{n} \mid n \in \mathbb{N}\right\}$ is an Ore subset of $A$.

This is applied to $A=R^{+}$and $\sigma_{i}=\left(\left\llcorner K_{i}^{-1}\right), \partial_{i}=\left(\left\llcorner X_{i}^{-}\right), i \in[1, r]\right.\right.$, recall (2.2). The skew derivations $\partial_{i}$ are regular because $\sigma_{i} \partial_{i} \sigma_{i}^{-1}=q^{-2} \partial_{i}, \forall i \in[1, r]$. Let $w s_{i_{1}} \ldots s_{i_{k}}=$ $w_{0}$, where $k=\ell\left(w_{0}\right)-\ell(w)$. Denote $w_{j}=w s_{i_{1}} \ldots s_{i_{j}}, j \in[0, k]$. It is easy to show by induction on $j$ that $\operatorname{deg}_{\partial_{i_{j}}} e_{w_{j}}^{\lambda}=-\left\langle w_{j} \lambda, \alpha_{i_{j}}^{\vee}\right\rangle, e_{w_{j-1}}^{\lambda}=t_{j} \partial_{i_{j-1}}^{-\left\langle w_{j} \lambda, \alpha_{i_{j}}^{\vee}\right\rangle}\left(e_{w_{j}}^{\lambda}\right)$ for some $t_{j} \in \mathbb{K}^{*}$, and that $\left\{\left(e_{w_{j}}^{\lambda}\right)^{n} \mid n \in \mathbb{N}\right\}$ is an Ore subset of $R^{+}$. This implies (2.7) and Lemma 2.1.

Denote the algebras

$$
R^{w}=R^{+}\left[E_{w}^{-1}\right], \quad w \in W
$$

and their invariant subalgebras with respect to the action of $\angle K_{i}, i \in[1, r]$, (cf. (2.2)):

$$
R_{0}^{w}=\left\{c_{\xi}^{\lambda}\left(e_{w}^{\lambda}\right)^{-1} \mid \lambda \in \mathcal{P}^{+}, \xi \in V(\lambda)^{*}\right\}
$$

One does not need to take span on the right-hand side of the above formula, see [20, equation (2)]. For $\mu=\lambda_{1}-\lambda_{2} \in \mathcal{P}, \lambda_{1}, \lambda_{2} \in \mathcal{P}^{+}$set

$$
e_{w}^{\mu}=e_{w}^{\lambda_{1}}\left(e_{w}^{\lambda_{2}}\right)^{-1} \in R^{+}\left[E_{w}^{-1}\right]
$$

It follows from (2.6) that this does not depend on the choice of $\lambda_{1}, \lambda_{2}$ and that $e_{w}^{\mu_{1}} e_{w}^{\mu_{2}}=$ $e_{w}^{\mu_{1}+\mu_{2}}$ for all $\mu_{1}, \mu_{2} \in \mathcal{P}$. For $y \in W$, define the quantum Schubert cell ideals of $R^{+}$

$$
Q(y)^{ \pm}=\operatorname{Span}\left\{c_{\xi}^{\lambda} \mid \lambda \in \mathcal{P}^{+}, \xi \in V(\lambda)^{*}, \xi \perp \mathcal{U}_{ \pm} T_{y} v_{\lambda}\right\}
$$

and the ideals

$$
Q(y)_{w}^{ \pm}=\left\{c_{\xi}^{\lambda} e_{w}^{-\lambda} \mid \lambda \in \mathcal{P}^{+}, \xi \in V(\lambda)^{*}, \xi \perp \mathcal{U}_{ \pm} T_{y} v_{\lambda}\right\}=Q(y)^{ \pm}\left(R^{+}\left[E_{w}^{-1}\right]\right) \cap R_{0}^{w}
$$

of $R_{0}^{w}$. Similar to (2.8), one does not need to take a span in $(2.10)$, see $[\mathbf{2 0}, \mathbf{3 8}]$. 
2.3. Quantum Schubert cell algebras. The algebras $\mathcal{U}_{ \pm}^{w}, w \in W$ were defined by De Concini et al. [9], and Lusztig [30, Section 40.2] as follows. Let

$$
w=s_{i_{1}} \ldots s_{i_{l}}
$$

be a reduced expression of $w$ (thus $l=\ell(w)$ ). Consider the roots

$$
\beta_{1}=\alpha_{i_{1}}, \beta_{2}=s_{i_{1}}\left(\alpha_{i_{2}}\right), \ldots, \beta_{l}=s_{i_{1}} \ldots s_{i_{l-1}}\left(\alpha_{i_{l}}\right)
$$

and the Lusztig root vectors

$$
X_{\beta_{1}}^{ \pm}=X_{i_{1}}^{ \pm}, X_{\beta_{2}}^{ \pm}=T_{s_{i_{1}}}\left(X_{i_{2}}^{ \pm}\right), \ldots, X_{\beta_{l}}^{ \pm}=T_{s_{i_{1}}} \ldots T_{s_{i_{l-1}}}\left(X_{i_{l}}^{ \pm}\right),
$$

see [30, Section 39.3]. By [9, Proposition 2.2] and [30, Proposition 40.2.1] the subalgebras $\mathcal{U}_{ \pm}^{w}$ of $\mathcal{U}_{ \pm}$generated by $X_{\beta_{j}}^{ \pm}, j \in[1, l]$ do not depend on the choice of the reduced decomposition of $w$ and have the Poincare-Birkhoff-Witt bases

$$
\left(X_{\beta_{l}}^{ \pm}\right)^{n_{l}} \ldots\left(X_{\beta_{1}}^{ \pm}\right)^{n_{1}}, \quad n_{1}, \ldots, n_{l} \in \mathbb{N} .
$$

The grading (2.3) induces $\mathcal{Q}$-gradings on the subalgebras $\mathcal{U}_{ \pm}^{w}$. The corresponding graded components will be denoted by $\left(\mathcal{U}_{ \pm}^{w}\right)_{\gamma}, \gamma \in \mathcal{Q}$. The algebra $R^{+}$is $\mathcal{P}$-graded by

$$
\text { wt } c_{\xi}^{\lambda}=v, \quad \lambda \in \mathcal{P}^{+}, v \in \mathcal{P}, \xi \in\left(V(\lambda)^{*}\right)_{\nu} .
$$

This induces $\mathcal{P}$-gradings on the algebras $R^{w}$ and $\mathcal{Q}$-gradings on the algebras $R_{0}^{w}$. The latter are given by

$$
\text { wt } c_{\xi}^{\lambda} e_{w}^{-\lambda}=v+w(\lambda), \quad \lambda \in \mathcal{P}^{+}, v \in \mathcal{P}, \xi \in\left(V(\lambda)^{*}\right)_{\nu} .
$$

For a given $\gamma \in \mathcal{Q}^{+} \backslash\{0\}$, let $m_{w}(\gamma)=\operatorname{dim}\left(\mathcal{U}_{+}^{w}\right)_{\gamma}=\operatorname{dim}\left(\mathcal{U}_{-}^{w}\right)_{-\gamma}$ and $\left\{u_{\gamma, i}\right\}_{i=1}^{m_{w}(\gamma)}$, $\left\{u_{-\gamma, i}\right\}_{i=1}^{m_{w}(\gamma)}$ be dual bases of $\left(\mathcal{U}_{+}^{w}\right)_{\gamma}$ and $\left(\mathcal{U}_{-}^{w}\right)_{-\gamma}$ with respect to the Rosso-Tanisaki form, see [24, Chap. 6]. The quantum $R$ matrix corresponding to $w$ is given by

$$
\mathcal{R}^{w}=1 \otimes 1+\sum_{\gamma \in \mathcal{Q}^{+}, \gamma \neq 0} \sum_{i=1}^{m_{w}(\gamma)} u_{\gamma, i} \otimes u_{-\gamma, i} \in \mathcal{U}_{+} \widehat{\otimes} \mathcal{U}_{-},
$$

where $\mathcal{U}_{+} \widehat{\otimes} \mathcal{U}_{-}$is the completion of $\mathcal{U}_{+} \otimes_{\mathbb{K}} \mathcal{U}_{-}$with respect to the descending filtration [30, Section 4.1.1]. Recall that there is a unique algebra anti-automorphism $\tau$ of $\mathcal{U}_{q}(\mathfrak{g})$ given by

$$
\tau\left(X_{i}^{ \pm}\right)=X_{i}^{ \pm}, \tau\left(K_{i}\right)=K_{i}^{-1}, \quad i=1, \ldots, r
$$

see [24, Lemma 4.6(b)]. It is graded with respect to (2.3) and satisfies

$$
\tau\left(T_{w} x\right)=T_{w^{-1}}^{-1}(\tau(x)), \quad \forall w \in W, x \in \mathcal{U}_{q}(\mathfrak{g}),
$$

see [24, equation 8.18(6)].

Proposition 2.2. ([40, Theorem 2.6]). Assume that $\mathbb{K}$ is an arbitrary base field, $q \in \mathbb{K}^{*}$ is not a root of unity and $\mathfrak{g}$ is a simple Lie algebra. For all Weyl group elements 
$w \in W$ the maps

$$
\begin{aligned}
& \phi_{w}^{+}: R_{0}^{w} \rightarrow \mathcal{U}_{-}^{w}, \quad \phi_{w}^{+}\left(c_{\xi}^{\lambda} e_{w}^{-\lambda}\right)=\left(c_{\xi, T_{w} v_{\lambda}}^{\lambda} \otimes \mathrm{id}\right)(\tau \otimes \mathrm{id}) \mathcal{R}^{w} \text { and } \\
& \phi_{w}^{-}: R_{0}^{w} \rightarrow T_{w}\left(\mathcal{U}_{+}^{w^{-1} w_{0}}\right), \quad \phi_{w}^{-}\left(e_{w}^{-\lambda} c_{\xi}^{\lambda}\right)=\left(\mathrm{id} \otimes c_{\xi, T_{w} v_{\lambda}}^{\lambda}\right) \mathcal{R}^{w},
\end{aligned}
$$

where $\lambda \in \mathcal{P}^{+}, \quad \xi \in V(\lambda)^{*}$, are well-defined surjective-graded algebra antihomomorphisms in the plus case and well-defined surjective-graded algebra homomorphisms in the minus case (with respect to the $\mathcal{Q}$-gradings (2.3) and (2.16)), recall (2.1). Their kernels are $\operatorname{ker} \phi_{w}^{ \pm}=Q(w)_{w}^{ \pm}$.

The plus case of this proposition was proved in [40, Theorem 2.6]. The minus case is analogous. In another form the plus case was obtained in [38, Theorem 3.7] for a version of $\mathcal{U}_{q}(\mathfrak{g})$ equpped with the opposite co-multiplication, and different braid group action and Lustig's root vectors. We note that in the plus and minus cases the term $e_{w}^{-\lambda}$ appears on different sides. This is because of the difference in the formulas for the co-products of $X_{i}^{ \pm}$. Moreover, the plus case requires the use of the anti-automorphism $\tau$, while the minus case does not. This is due to the fact that one constructs dual bases of $\mathcal{U}_{ \pm}$with respect to the Rosso-Tanisaki form by multiplying Lusztig's root vectors in the opposite order, see e.g. [24, equations 8.30(1) and (2)].

2.4. Two-cocycle twists. Let $C$ be an (additive) abelian group and $R$ be a $C$-graded $\mathbb{K}$-algebra. Following Artin et al. [1], for a 2-cocycle $\mathbf{p} \in Z^{2}\left(C, \mathbb{K}^{*}\right)$, define a new algebra structure on $R$ by twisting the multiplication of $R$ by

$$
b_{1} * b_{2}=\mathbf{p}\left(\alpha_{1}, \alpha_{2}\right) b_{1} b_{2}, \quad \alpha_{i} \in C, b_{i} \in R_{\alpha_{i}}, i=1,2 .
$$

This algebra will be denoted by $R_{\mathbf{p}}$. Up to an isomorphism $R_{\mathbf{p}}$ depends only on the cohomology class of $\mathbf{p}$, see [1, Section 3]. Thus, we can assume that the 2cocycle $\mathbf{p}$ is normalized by $\mathbf{p}(0,0)=1$. The group of such cocycles will be denoted by $Z^{2}\left(C, \mathbb{K}^{*}\right)_{n}$. The normalization $\mathbf{p}(0,0)=1$ implies that for all $\alpha \in C, \mathbf{p}(\alpha, 0)=$ $\mathbf{p}(0, \alpha)=1$. Therefore, the multiplications $R_{0} \times R_{\alpha} \rightarrow R_{\alpha}, R_{\alpha} \times R_{0} \rightarrow R_{\alpha}$ remain undeformed for all $\alpha \in C$ and the multiplicative identity in $R$ is an identity of $R_{\mathbf{p}}$. Define

$$
\mathbf{r}(\alpha, \beta)=\mathbf{p}(\alpha, \beta) \mathbf{p}(\beta, \alpha)^{-1}, \quad \alpha, \beta \in C .
$$

By [1, Proposition 1], if $C$ is a free abelian group, then

$$
\mathbf{r}: C \times C \rightarrow \mathbb{K}^{*} \text { is a bi-character, }
$$

which is clearly multiplicatively skew symmetric in the sense that $\mathbf{r}(\beta, \alpha)=\mathbf{r}(\alpha, \beta)^{-1}$, $\forall \alpha, \beta \in C$.

For $w \in W$, denote the support of $w$ :

$$
\mathcal{S}(w)=\left\{i \in[1, r] \mid s_{i} \leq w\right\}=\left\{i \in[1, r] \mid s_{i} \text { appears in a reduced expression of } w\right\} .
$$

For $I \subseteq[1, r]$, denote $\mathcal{Q}_{I}=\oplus_{i \in I} \mathbb{Z} \alpha_{i}, \mathcal{P}_{I}=\oplus_{i \in I} \mathbb{Z} \omega_{i}, \mathcal{Q}_{I}^{+}=\mathcal{Q}_{I} \cap \mathcal{Q}^{+}$and $\mathcal{P}_{I}^{+}=\mathcal{P}_{I} \cap$ $\mathcal{P}^{+}$. Then

$$
[1, r] \backslash \mathcal{S}(w)=\left\{i \in[1, r] \mid w\left(\omega_{i}\right)=\omega_{i}\right\},
$$

see [40, Lemma 3.2 and equation (3.2)]. We have the following. 
LEMma 2.3. ([40, Lemma 3.2(ii), equation (2.43)]). The subgroup of $\mathcal{Q}$ generated by all $\gamma$ such that $\left(\mathcal{U}_{ \pm}^{w}\right)_{\gamma} \neq 0$ is equal to $\mathcal{Q}_{\mathcal{S}(w)}$.

For $\mathbf{p} \in Z^{2}\left(\mathcal{Q}_{\mathcal{S}(w)}, \mathbb{K}^{*}\right)_{n}$ define the multi-parameter quantum Schubert cell algebra

$$
\mathcal{U}_{ \pm, \mathbf{p}}^{w}=\left(\mathcal{U}_{ \pm}^{w}\right)_{\mathbf{p}}
$$

For $\gamma \in \mathcal{Q}_{\mathcal{S}(w)}$, define the character of $\mathbb{T}^{|\mathcal{S}(w)|}$

$$
t \mapsto t^{\gamma}=\prod_{i \in \mathcal{S}(w)} t_{i}^{\left\langle\gamma, \omega_{i}\right\rangle}, \quad t=\left(t_{i}\right)_{i \in \mathcal{S}(w)} \in \mathbb{T}^{|\mathcal{S}(w)|}
$$

and consider the rational $\mathbb{T}^{|\mathcal{S}(w)|}$-action on $\mathcal{U}_{ \pm}^{w}$ by algebra automorphisms

$$
t . x=t^{\gamma} x, \quad t \in \mathbb{T}^{|\mathcal{S}(w)|}, x \in\left(\mathcal{U}_{ \pm}^{w}\right)_{\gamma}, \gamma \in \mathcal{Q}_{\mathcal{S}(w)} .
$$

Since the twists $\mathcal{U}_{ \pm, \mathbf{p}}^{w}$ are graded, for all $\mathbf{p} \in Z^{2}\left(\mathcal{Q}_{\mathcal{S}(w)}, \mathbb{K}^{*}\right)_{n},(2.23)$ induces a rational $\mathbb{T}^{|\mathcal{S}(w)|}$-action on $\mathcal{U}_{ \pm, \mathbf{p}}^{w}$ by algebra automorphisms.

For the simplicity of the notation, define

$$
b_{w, \xi}^{\lambda}=\phi_{w}^{+}\left(c_{\xi}^{\lambda} e_{w}^{-\lambda}\right), a_{y, w}^{\lambda}=\phi_{w}^{+}\left(e_{y}^{\lambda} e_{w}^{-\lambda}\right) \in \mathcal{U}_{-}^{w}, \quad y \leq w \in W, \lambda \in \mathcal{P}^{+}, \xi \in V(\lambda)^{*}
$$

and denote by $x y:=x * y$ the multiplication in $\mathcal{U}_{ \pm, \mathbf{p}}^{w}$. Equation (2.21) implies that $w(\mu)=y(\mu)=\mu$ for all $\mu \in \mathcal{P}_{[1, r] \backslash \mathcal{S}(w)}, y \leq w \in W$, and from (2.6) we obtain that

$$
a_{y, w}^{\lambda+\lambda^{\prime}}=s a_{y, w}^{\lambda}, \quad \forall \lambda \in \mathcal{P}_{\mathcal{S}(w)}^{+}, \lambda^{\prime} \in \mathcal{P}_{[1, r] \backslash \mathcal{S}(w)}^{+}, y \leq w \in W,
$$

for some $s \in \mathbb{K}^{*}$. Recall Proposition 2.2 and (2.24). Equation (2.4) implies the following commutation relation in $\mathcal{U}_{-, \mathbf{p}}^{w}$ :

$$
\begin{aligned}
& \mathbf{p}\left(w \lambda_{2}+v_{2}, w \lambda_{1}+v_{1}\right)^{-1} b_{w, \xi_{2}}^{\lambda_{2}} b_{w, \xi_{1}}^{\lambda_{1}}=\mathbf{p}\left(w \lambda_{1}+v_{1}, w \lambda_{2}+v_{2}\right)^{-1} q^{\left\langle w \lambda_{1}-v_{1}, w \lambda_{2}+v_{2}\right\rangle} b_{w, \xi_{1}}^{\lambda_{1}} b_{w, \xi_{2}}^{\lambda_{2}} \\
& \quad+\sum_{\gamma \in \mathcal{Q}^{+}, \gamma \neq 0} \sum_{i=1}^{m(\gamma)} \mathbf{p}\left(w \lambda_{1}+v_{1}-\gamma, w \lambda_{2}+v_{2}+\gamma\right)^{-1} q^{\left\langle w \lambda_{1}-v_{1}+\gamma, w \lambda_{2}+v_{2}+\gamma\right\rangle-\left\langle\gamma, w \lambda_{2}\right\rangle} \\
& \quad \times b_{w, S^{-1}\left(u_{-\gamma, i}\right) \xi_{1}}^{\lambda_{1}} b_{w, S^{-1}\left(u_{\gamma, i}\right) \xi_{2}}^{\lambda_{2}}
\end{aligned}
$$

for all $\lambda_{i} \in \mathcal{P}^{+}, v_{i} \in-w \lambda_{i}+\mathcal{Q}_{\mathcal{S}(w)}$ and $\xi_{i} \in\left(V\left(\lambda_{i}\right)^{*}\right)_{\nu_{i}}$. The elements $u_{ \pm \gamma, i} \in\left(\mathcal{U}_{ \pm}\right)_{ \pm \gamma}$ are as in (2.4). For the values of $\gamma \in \mathcal{Q}^{+}$, for which $w \lambda_{2}+v_{2}+\gamma \notin \mathcal{Q}(\mathcal{S}(w))$ or $w \lambda_{1}+v_{1}-\gamma \notin \mathcal{Q}(\mathcal{S}(w))$, i.e. $\mathbf{p}\left(w \lambda_{2}+v_{2}+\gamma, w \lambda_{1}+v_{1}-\gamma\right)$ is not defined, the term $b_{w, S^{-1}\left(u_{-\gamma, i}\right) \xi_{1}}^{\lambda_{1}} b_{w, S^{-1}\left(u_{\gamma, i}\right) \xi_{2}}^{\lambda_{2}}$ vanishes by Proposition 2.2 and Lemma 2.3. The related product in (2.26) is set to be equal to 0 .

The Levendorskii-Soibelman straightening law (see, for instance, [4, Proposition I.6.10] and [40, equation (2.38)]) is the following straightening law in $\mathcal{U}_{-, \mathbf{p}}^{w}$ :

$$
\begin{aligned}
& X_{\beta_{j}}^{-} X_{\beta_{i}}^{-}-\mathbf{r}\left(\beta_{j}, \beta_{i}\right) q^{-\left\langle\beta_{j}, \beta_{i}\right\rangle} X_{\beta_{i}}^{-} X_{\beta_{j}}^{-} \\
& =\sum_{\mathbf{k}=\left(k_{i+1}, \ldots, k_{j-1}\right) \in \mathbb{N} \times(j-i-2)} s_{\mathbf{k}}\left(X_{\beta_{j-1}}^{-}\right)^{k_{j-1}} \ldots\left(X_{\beta_{i+1}}^{-}\right)^{k_{i+1}}, \quad s_{\mathbf{k}} \in \mathbb{K}
\end{aligned}
$$

for all $i<j$, recall (2.19). Fix a reduced expression of $w$ as in (2.11). For $j \in[0, l]$ denote $w_{j}=s_{i_{1}} \ldots s_{i_{j}}$ (thus $w_{0}=1$ and $w_{l}=w$ ). By abuse of notation we will denote by the 
same symbols the restrictions of $\mathbf{p}$ and $\mathbf{r}$ to $\mathcal{Q}_{\mathcal{S}\left(w_{j}\right)} \times \mathcal{Q}_{\mathcal{S}\left(w_{j}\right)}$. Because of (2.20), for all $j \in[1, l]$, there exists a unique $t_{j} \in \mathbb{T}^{\left|\mathcal{S}\left(w_{j}\right)\right|}$ such that

$$
t_{j}^{\beta_{i}}=\mathbf{r}\left(\beta_{j}, \beta_{i}\right) q^{-\left\langle\beta_{j}, \beta_{i}\right\rangle}, \quad \forall i \leq j,
$$

recall (2.22). The following lemma is a direct consequence of (2.14), (2.27) and (2.28).

Lemma 2.4. For all base fields $\mathbb{K}, q \in \mathbb{K}^{*}$ not a root of unity, Weyl group elements $w \in W$, reduced expressions (2.11), and 2-cocycles $\mathbf{p} \in Z^{2}\left(\mathcal{Q}_{\mathcal{S}(w)}, \mathbb{K}^{*}\right)_{n}$ we have:

(a) The subalgebra of $\mathcal{U}_{-, \mathbf{p}}^{w}$ generated by $X_{\beta_{1}}^{-}, \ldots, X_{\beta_{j}}^{-}$is isomorphic to $\mathcal{U}_{-, \mathbf{p}}^{w_{j}}, j \in[1, l]$.

(b) The algebra $\mathcal{U}_{-, \mathbf{p}}^{w_{j}}$ is isomorphic to the Ore extension $\mathcal{U}_{-, \mathbf{p}}^{w_{j-1}}\left[x,\left(t_{j} \cdot\right), \delta_{j}\right]$ for some (left) $\left(t_{j} \cdot\right)$-skew derivation $\delta_{j}$ of $\mathcal{U}_{-, \mathbf{p}}^{w_{j-1}}$ satisfying $\left(t_{j} \cdot\right) \delta_{j}=q^{-\left\langle\beta_{j}, \beta_{j}\right\rangle} \delta_{j}\left(t_{j} \cdot\right), j \in[1, l]$. Here $\left(t_{j} \cdot\right)$ denotes the restriction to $\mathcal{U}_{-, \mathbf{p}}^{w_{j-1}}$ of the automorphism of $\mathcal{U}_{-, \mathbf{p}}^{w_{j}}$ induced from the action (2.23), and $\mathcal{U}_{-, \mathbf{p}}^{1}=\mathbb{K}$.

(c) The eigenvalues $t_{j} \cdot X_{\beta_{j}}^{-}=q^{-\left\langle\beta_{j}, \beta_{j}\right\rangle} X_{\beta_{j}}^{-}$are not roots of unity.

Since the algebras $\mathcal{U}_{-, \mathbf{p}}^{w}$ are iterated Ore extensions, they are noetherian, and the Brown-Goodearl theorem [4, Proposition II.2.9] implies that each $\mathbb{T}^{|\mathcal{S}(w)|}$-prime ideal of $\mathcal{U}_{-, \mathbf{p}}^{w}$ with respect to the action $(2.23)$ is prime.

\section{A description of the spectra of $\mathcal{U}_{-, \mathrm{p}}^{w}$.}

3.1. Statement of the main result. In this section we describe an explicit stratification of $\operatorname{Spec} \mathcal{U}_{-, \mathbf{p}}^{w}$ by the spectra of commutative Laurent polynomial rings over $\mathbb{K}$ and the structure of the poset of $\mathbb{T}^{|\mathcal{S}(w)|}$-invariant prime ideals of $\mathcal{U}_{-, \mathbf{p}}^{w}$ for the action (2.23). For $y \in W^{\leq w}$, define the ideals

$$
I_{w}(y)=\phi_{w}^{+}\left(Q(w)_{w}^{+}+Q(y)_{w}^{-}\right)=\phi_{w}^{+}\left(Q(y)_{w}^{-}\right)
$$

of $\mathcal{U}_{-, \mathbf{p}}^{w}$ and denote

$$
A_{y, w}=\mathbb{K}^{*}\left\{a_{y, w}^{\lambda} \mid \lambda \in \mathcal{P}^{+}\right\}=\mathbb{K}^{*}\left\{a_{y, w}^{\lambda} \mid \lambda \in \mathcal{P}_{\mathcal{S}(w)}^{+}\right\},
$$

cf. (2.24)-(2.25). By (2.6) and (2.26), $A_{y, w}$ is a multiplicative subset of $\mathcal{U}_{-, \mathrm{p}}^{w}$ consisting of elements which are normal modulo $I_{w}(y)$ :

$$
a_{y, w}^{\lambda} x=\mathbf{r}((w-y) \lambda, \gamma) q^{-\langle(w+y) \lambda, \gamma\rangle} x a_{y, w}^{\lambda} \bmod I_{w}(y),
$$

$\forall \lambda \in \mathcal{P}^{+}, \gamma \in \mathcal{Q}_{\mathcal{S}(w)}, x \in\left(\mathcal{U}_{-, \mathbf{p}}\right)_{\gamma}$. The following theorem describes Spec $\mathcal{U}_{-, \mathbf{p}}^{w}$ set theoretically and provides some information for its Zariski topology.

THEOREM 3.1. Assume that $\mathbb{K}$ is an arbitrary field, $q \in \mathbb{K}^{*}$ is not a root of unity, $\mathfrak{g}$ is a simple Lie algebra, $w$ is an element of the corresponding Weyl group $W$ and $\mathbf{p} \in Z^{2}\left(\mathcal{Q}_{\mathcal{S}(w)}, \mathbb{K}^{*}\right)_{n}$ is a normalized cocycle. Then:

(a) The $\mathbb{T}^{|\mathcal{S}(w)|}$-invariant prime ideals of $\mathcal{U}_{-, \mathbf{p}}^{w}$ are the ideals $I_{w}(y)$ for all $y \in W \leq w$. The map $y \in W^{\leq w} \mapsto I_{w}(y) \in \mathbb{T}^{|\mathcal{S}(w)|}$-Spec $\mathcal{U}_{-, \mathbf{p}}^{w}$ is an isomorphism of posets with respect to the Bruhat order and the inclusion order on ideals. In particular, ideals $I_{w}(y)$ are distinct.

(b) For all $y \in W^{\leq w}, \quad I_{w}(y) \cap A_{y, w}=\emptyset$ and the quotient ring $R_{y, w}=$ $\left(\mathcal{U}_{-, \mathbf{p}}^{w} / I_{w}(y)\right)\left[A_{y, w}^{-1}\right]$ is a $\mathbb{T}^{|\mathcal{S}(w)|}$-simple domain. 
(c) For every prime ideal $J$ of $\mathcal{U}_{-, \mathbf{p}}^{w}$ there exists a unique $y \in W^{\leq w}$ such that

$$
J \supseteq I_{w}(y) \text { and } J \cap A_{y, w}=\emptyset .
$$

Denote the corresponding subset of $\operatorname{Spec}_{-, \mathbf{p}}^{w}$ by $\operatorname{Spec}_{I_{w}(y)} \mathcal{U}_{-, \mathbf{p}}^{w}$, so

$$
\operatorname{Spec}_{-, \mathbf{p}}^{w}=\bigsqcup_{y \in W \leq w} \operatorname{Spec}_{I_{w}(y)} \mathcal{U}_{-, \mathbf{p}}^{w}
$$

(d) The centre $Z\left(R_{y, w}\right)$ is a Laurent polynomial ring over $\mathbb{K}$ and we have a homeomorphism

$$
\iota_{y}: \operatorname{Spec} Z\left(R_{y, w}\right) \rightarrow \operatorname{Spec}_{I_{w}(y)} \mathcal{U}_{-, \mathbf{p}}^{w},
$$

where for $J_{0} \in \operatorname{Spec} Z\left(R_{y, w}\right), \quad \iota_{y}\left(J_{0}\right)$ is the ideal of $\mathcal{U}_{-, \mathbf{p}}^{w}$ containing $I_{w}(y)$ such that $\iota_{y}\left(J_{0}\right) / I_{w}(y)=J_{0} R_{y, w} \cap\left(\mathcal{U}_{-, \mathbf{p}}^{w} / I_{w}(y)\right)$. The Zariski closures of the strata $\operatorname{Spec}_{I_{w}(y)} \mathcal{U}_{-, \mathbf{p}}^{w}$ are given by

$$
\overline{\operatorname{Spec}_{I_{w}(y)} \mathcal{U}_{-, \mathbf{p}}^{w}}=\bigsqcup_{y^{\prime} \in W \leq y} \operatorname{Spec}_{I_{w}\left(y^{\prime}\right)} \mathcal{U}_{-, \mathbf{p}}^{w}
$$

Gorelik described in [20] the torus invariant prime ideals of the algebras $R_{0}^{w}$ and the resulting stratification of $\operatorname{Spec} R_{0}^{w}$ as in Theorem 3.1 under the condition that $\mathbb{K}=k(q)$ for a field $k$ of characteristic 0 . Via the homomorphism from Proposition 2.2 , this establishes Theorem 3.1 in the untwisted case under those assumptions on $\mathbb{K}$ and $q$. We show that Gorelik's arguments work under the weaker assumptions on $\mathbb{K}$ and $q$, taking shortcuts at some steps using different arguments based on the results of Goodearl and Letzter [18] and Proposition 2.2. The untwisted case of Theorem 3.1(a) was stated in [41, Theorem 3.1] but the proof was not given there. The proof of Theorem 3.1 is given in Section 4 and Sections $4-4$ contain some preparatory results.

Consider a $\mathbb{K}$-algebra $R$ equipped with a rational action of a $\mathbb{K}$-torus $\mathbb{T}^{r}$ by algebra automorphisms. It gives rise to a $\mathbb{Z}^{r}$-grading of $R$. Then for each $\mathbf{p} \in Z^{2}\left(\mathbb{Z}^{n}\right.$, $\left.\mathbb{K}^{*}\right)$ the sets of $\mathbb{T}^{r}$-prime ideals of $R$ and $R_{\mathbf{p}}$ coincide. (This is similar to the observation of Goodearl and Lenagan for twists of completely prime ideals in the proof of [15, Theorem 4.1].) In particular, $R$ is ${\mathbb{\mathbb { V } ^ { r }}}^{r}$-simple if and only if $R_{\mathbf{p}}$ is $\mathbb{\mathbb { T }}^{r}$-simple. Using [18, Lemma 6.3(c) and Corollary 6.5] and [4, Theorem II.6.4] one can show that the twisted case of Theorem 3.1 can be deduced from the untwisted case. For most of our proofs of Theorem 3.1 we directly work with the twisted case, since it does not make a difference in the proofs.

It follows from Theorem 3.1(c)-(d) that $\operatorname{Spec}_{I_{w}(y)} \mathcal{U}_{-, \mathbf{p}}^{w}$ are precisely the GoodearlLetzter strata [18] of $\operatorname{Spec} \mathcal{U}_{-, \mathbf{p}}^{w}$. Namely, we have that

$$
\operatorname{Spec}_{I_{w}(y)} \mathcal{U}_{-, \mathbf{p}}^{w}=\left\{J \in \operatorname{Spec}_{\mathcal{U}_{-, \mathbf{p}}^{w}}^{w} \mid \cap_{t \in \mathbb{T}|\mathcal{S}(w)|} t . J=I_{w}(y)\right\} .
$$

REMARK 3.2. For $I \subseteq[1, r]$

$$
R_{I}^{+}=\operatorname{Span}\left\{c_{\xi}^{\lambda} \mid \lambda \in \mathcal{P}_{I}^{+}, \xi \in V(\lambda)^{*}\right\} \subseteq R^{+}
$$

is a subalgebra of $R^{+}$(called the quantum partial flag variety associated to $I$ ), see [37]. Joseph's argument for the proof of Lemma 2.1 shows that $E_{\mathcal{S}(w), w}=\left\{e_{w}^{\lambda} \mid \lambda \in \mathcal{P}_{\mathcal{S}(w)}^{+}\right\}$is 
an Ore subset of $R_{\mathcal{S}(w)}^{+}$. Define the subalgebra

$$
R_{\mathcal{S}(w), 0}^{w}=\left\{c_{\xi}^{\lambda}\left(e_{w}^{\lambda}\right)^{-1} \mid \lambda \in \mathcal{P}_{\mathcal{S}(w)}^{+}, \xi \in V(\lambda)^{*}\right\} \subset R_{\mathcal{S}(w)}^{+}\left[E_{\mathcal{S}(w), w}^{-1}\right]
$$

and its ideals

$$
Q(y)_{\mathcal{S}(w), w}^{ \pm}=\left\{c_{w, \xi}^{\lambda}\left(e_{w}^{\lambda}\right)^{-1} \mid \lambda \in \mathcal{P}_{\mathcal{S}(w)}^{+}, \xi \in V(\lambda)^{*}, \xi \perp \mathcal{U}_{ \pm} T_{y} v_{\lambda}\right\}
$$

The argument of the proof of [40, Theorem 2.6] gives that the restriction

$$
\left.\phi_{+}^{w}\right|_{R_{\mathcal{S}(w), 0}^{w}}: R_{\mathcal{S}(w), 0}^{w} \rightarrow \mathcal{U}_{-}^{w}
$$

is a surjective algebra anti-homomorphism with kernel $Q(w)_{\mathcal{S}(w), w}^{+}$, see also [37, Theorem 3.6]. The proof of Theorem 3.1 works in the same way if the map $\phi_{w}^{+}$is substituted with its restriction $\left.\phi_{w}^{+}\right|_{R_{\mathcal{S}(w), 0}^{w}}$ and the $\mathbb{T}^{|\mathcal{S}(w)|}$-primes of $\mathcal{U}_{-}^{w}$ are also given by

$$
I_{w}(y)=\phi_{+}^{w}\left(Q(y)_{\mathcal{S}(w), w}^{-}\right), \quad \forall y \in W^{\leq w} .
$$

We finish with noting that [17, Theorem 2.3] of Goodearl and Letzter, and Lemma 2.4 imply at once the following.

Proposition 3.3. Every prime ideal of $\mathcal{U}_{-, \mathbf{p}}^{w}$ is completely prime for all base fields $\mathbb{K}, q \in \mathbb{K}^{*}$ not a root of unity, Weyl group elements $w \in W$ and 2-cocycles $\mathbf{p} \in Z^{2}\left(\mathcal{Q}_{\mathcal{S}(w)}, \mathbb{K}^{*}\right)_{n}$ such that the subgroup of $\mathbb{K}^{*}$ generated by

$$
\mathbf{r}\left(\beta_{i}, \beta_{j}\right) q^{\left\langle\beta_{i}, \beta_{j}\right\rangle}=\left(\mathbf{r}\left(\beta_{j}, \beta_{i}\right) q^{-\left\langle\beta_{j}, \beta_{i}\right\rangle}\right)^{-1}, \quad 1 \leq i<j \leq \ell(w)
$$

is torsion free, recall (2.12).

3.2. Complete primeness of certain ideals. We start with a couple of auxiliary results for the proof of Theorem 3.1. The following lemma was proved by Joseph for $\mathbb{K}=\mathbb{C}, q \in \mathbb{C}^{*}$ not a root of unity [27, Proposition 10.1.8] and for $\mathbb{K}=k(q)$, char $k=0$, [26, Proposition 7.3]. It is easy to verify that Joseph's proof works in the general case. We provide the second proof based on Proposition 2.2.

LemMA 3.4. Let $\mathbb{K}$ be an arbitrary field, $q \in \mathbb{K}^{*}$ not a root of unity. The ideals $Q(w)^{ \pm}$ and $Q(y)_{w}^{ \pm}$of $R^{+}$and $R_{0}^{w}$ are completely prime for all $w \in W, y \in W^{\leq w}$ in the plus case and $y \in W^{\geq w}$ in the minus case.

Proof. By Lemma 2.4(b) $\mathcal{U}_{ \pm}^{w}$ is a domain, $\forall w \in W$. Proposition 2.2 implies that $Q(w)_{w}^{ \pm}=\operatorname{ker} \phi_{w}^{ \pm}$is a completely prime ideal of $R_{0}^{w}$. It is straightforward to verify that the map

$$
\begin{aligned}
& \left(R_{0}^{w} / Q(w)_{w}^{ \pm}\right) \# \mathbb{K}[\mathcal{P}] \rightarrow\left(R^{+}\left[E_{w}^{-1}\right] /\left(Q(w)^{ \pm} R^{+}\left[E_{w}^{-1}\right]\right),\right. \\
& \quad\left(c_{\xi}^{\lambda} e_{w}^{-\lambda}+Q(w)_{w}^{ \pm}\right) \# \mu \mapsto c_{\xi}^{\lambda} e_{w}^{-\lambda+\mu}+Q(w)^{ \pm} R^{+}\left[E_{w}^{-1}\right], \quad \mu \in \mathcal{P}, \lambda \in \mathcal{P}^{+}, \xi \in V(\lambda)^{*}
\end{aligned}
$$

is (a well-defined) algebra isomorphism, where the smash product is defined via the action

$$
\mu \cdot\left(r+Q(w)_{w}^{ \pm}\right)=q^{\mp\langle w \mu, \gamma\rangle}\left(r+Q(w)_{w}^{ \pm}\right), \quad \mu \in \mathcal{P}, r \in\left(R_{0}^{w}\right)_{\gamma}, \gamma \in \mathcal{Q},
$$


cf. (2.4) and (2.16). Therefore, $Q(w)^{ \pm} R^{+}\left[E_{w}^{-1}\right]$ is a completely prime ideal of $R^{+}\left[E_{w}^{-1}\right]$. It follows that $Q(w)^{ \pm}=Q(w)^{ \pm} R^{+}\left[E_{w}^{-1}\right] \cap R^{+}$is a completely prime ideal of $R^{+}, \forall w \in W$.

We have $T_{w} v_{\lambda} \in \mathcal{U}_{ \pm} T_{y} v_{\lambda}$, for all $\lambda \in \mathcal{P}^{+}$and $y \in W^{\geq w}$ in the plus case, $y \in W^{\leq w}$ in the minus case, see [27, Lemma 4.4.3]. Therefore, in those cases $Q(y)^{ \pm} \cap E_{w}=$ $\emptyset$ and thus $Q(y)^{ \pm} R^{+}\left[E_{w}^{-1}\right]$ is a completely prime ideal of $R^{+}\left[E_{w}^{-1}\right]$. So $Q(y)_{w}^{ \pm}=$ $Q(y)^{ \pm} R^{+}\left[E_{w}^{-1}\right] \cap R_{0}^{w}$ is a completely prime ideal of $R_{0}^{w}$.

LEMMA 3.5. For all base fields $\mathbb{K}, q \in \mathbb{K}^{*}$ not a root of unity, Weyl group elements $y \leq w$ and 2-cocycles $\mathbf{p} \in Z^{2}\left(\mathcal{Q}_{\mathcal{S}(w)}, \mathbb{K}^{*}\right)_{n}$ :

(a) $Q(w)_{w}^{+}+Q(y)_{w}^{-}$is a completely prime ideal of $R_{0}^{w}$ and

$$
\left(Q(w)_{w}^{+}+Q(y)_{w}^{-}\right) \cap\left\{e_{y}^{\lambda} e_{w}^{-\lambda} \mid \lambda \in \mathcal{P}_{\mathcal{S}(w)}^{+}\right\}=\emptyset,
$$

(b) $I_{w}(y)=\phi_{w}^{+}\left(Q(w)_{w}^{+}+Q(y)_{w}^{-}\right)$is a completely prime ideal of $\mathcal{U}_{-, \mathbf{p}}^{w}$ and $I_{w}(y) \cap$ $A_{y, w}=\emptyset$.

Assume in the setting of Section 3 that $C$ is a finite rank free abelian group. Then for a graded subspace $I$ of $R, I$ is a completely prime ideal of $R_{\mathrm{p}}$ if and only if $I$ is a completely prime ideal of $R$, see the proof of [15, Theorem 4.1]. Therefore, it is sufficient to prove only the untwisted case of Lemma 3.5(a). Taking into account Proposition 2.2, we see that part (b) of Lemma 3.5 follows from the first part of the lemma.

Gorelik [20, Lemma 6.6] stated the untwisted case of Lemma 3.5(a) for $\mathbb{K}=k(q)$, char $k=0$. Her proof works under the above more general assumptions on $\mathbb{K}$ and $q$. We review the key steps of her proof below to show this. For $\mu \in \mathcal{P}$ denote the automorphism

$$
\psi_{w}^{\mu}(r)=c_{w}^{-\mu} r c_{w}^{\mu}, \quad r \in R^{w}
$$

of $R^{w}$. It is obvious that $R_{0}^{w}$ is stable under it. Recall the skew derivations $\partial_{i}=\left(\left\llcorner X_{i}^{-}\right)\right.$ of $R^{+}$from Section 2. By a direct commutation argument, one shows that for all $w \in W$, $i \in[1, r]$ such that $s_{i} w<w, \lambda \in \mathcal{P}^{+}, \mu, v \in \mathcal{P}, \xi \in\left(V(\lambda)^{*}\right)_{v}$ and $t$ in the algebraic closure of $\mathbb{K}$ :

$$
\begin{aligned}
& \exists k \in \mathbb{Z}_{+} \text {such that }\left(\psi_{w}^{\mu}-t . \mathrm{id}\right)^{k} c_{\xi}^{\lambda}=0 \Rightarrow \\
& \qquad \exists \in \mathbb{Z}_{+} \text {such that }\left(\psi_{w}^{\mu}-t q^{-\langle v, w \mu\rangle+\left\langle\nu-m \alpha_{i}, s_{i} w \mu\right\rangle} \mathrm{id}\right)^{k}\left(\partial_{i}^{m} c_{\xi}^{\lambda}\right)=0,
\end{aligned}
$$

where $m \in \mathbb{N}$ denotes the $\partial_{i}$-degree of $c_{\xi}^{\lambda}$. For $r \in R^{w}$ set

$$
\mathrm{wt}_{w}(r)=\gamma \in \mathcal{Q} \text { if }\left(\psi_{w}^{\mu}-q^{\langle\gamma, \mu\rangle} \mathrm{id}\right)^{k} r=0 \text { for some } k \in \mathbb{Z}_{+} \text {and all } \mu \in \mathcal{P} .
$$

We say that $\xi \in V(\lambda)^{*}$ is homogeneous if $\xi \in\left(V(\lambda)^{*}\right)_{v}$ for some $v \in \mathcal{P}$. For a reduced expression $v=s_{i_{1}} \ldots s_{i_{k}} \in W$ and a homogeneous element $\xi \in V(\lambda)^{*}$, denote

$$
\partial_{v}^{*}\left(c_{\xi}^{\lambda}\right):=\partial_{i_{1}}^{m_{1}} \ldots \partial_{i_{k}}^{m_{k}}\left(c_{\xi}^{\lambda}\right)
$$

where for $j=k, \ldots, 1, m_{j}$ is the $\partial_{i_{j}}$-degree of $\partial_{i_{j+1}}^{m_{j+1}} \ldots \partial_{i_{k}}^{m_{i_{k}}}\left(c_{\xi}^{\lambda}\right)$, see Section 2. By induction (3.8) implies that for all homogeneous $\xi \in V(\lambda)$, if $\mathrm{wt}_{w}\left(c_{\xi}^{\lambda} e_{w}^{-\lambda}\right)$ exists, then

$$
\begin{aligned}
& \mathrm{wt}_{w}\left(c_{\xi}^{\lambda} e_{w}^{-\lambda}\right)-w^{-1} \operatorname{wt}\left(c_{\xi}^{\lambda} e_{w}^{-\lambda}\right) \\
& \quad=\mathrm{wt}_{1}\left(\left(\partial_{w^{-1}}^{*}\left(c_{\xi}^{\lambda}\right)\right) e_{1}^{-\lambda}\right)-\mathrm{wt}\left(\left(\partial_{w^{-1}}^{*}\left(c_{\xi}^{\lambda}\right)\right) e_{1}^{-\lambda}\right)=-2 \mathrm{wt}\left(\left(\partial_{w^{-1}}^{*}\left(c_{\xi}^{\lambda}\right)\right) e_{1}^{-\lambda}\right),
\end{aligned}
$$


recall (2.6) and (2.16). The last equality follows from $e_{1}^{-\mu} c_{\xi}^{\lambda} e_{1}^{\mu}=q^{-\langle\mu, \lambda+\nu\rangle} c_{\xi}^{\lambda}, \forall \lambda \in \mathcal{P}^{+}$, $\mu, v \in \mathcal{P}, \xi \in\left(V(\lambda)^{*}\right)_{v}$, which is a special case of (2.4). The automorphisms $\psi_{w}^{\mu}, \mu \in \mathcal{P}$ of $R_{0}^{w}$ commute, because of (2.6) and act locally finitely, since they preserve the grading (2.16). Therefore, $R_{0}^{w}$ is a direct sum of the common generalized $\psi_{w}^{\mu}$-eigenspaces, $\mu \in \mathcal{P}$. Equations (3.8) and (3.10), and the fact that for each $\gamma \in \mathcal{Q}$ there exists $\lambda \in \mathcal{P}^{+}$such that $\left(R_{0}^{w}\right)_{\gamma}=\left\{c_{\xi}^{\lambda} e_{w}^{-\lambda} \mid \xi \in\left(V(\lambda)^{*}\right)_{\gamma-w \lambda}\right\}$ imply that

$$
R_{0}^{w}=\bigoplus_{\gamma \in-2 \mathcal{Q}^{+}} R_{0}^{w}[\gamma],
$$

where $R_{0}^{w}[\gamma]$ is the span of all homogeneous elements $r \in R_{0}^{w}$ for which $\mathrm{wt}_{w}(r)$ exists and

$$
\mathrm{wt}_{w}(r)-w^{-1} \mathrm{wt}(r)=\gamma, \quad \gamma \in-2 \mathcal{Q}^{+} .
$$

It follows from (2.4) that

$$
\psi_{w}^{\mu}(r)=q^{\left\langle\mu, w^{-1} \gamma^{\prime}\right\rangle} r \quad \bmod Q(w)_{w}^{+}, \quad \forall \gamma^{\prime} \in \mathcal{Q}, r \in\left(R_{0}^{w}\right)_{\gamma^{\prime}}
$$

Thus, $\oplus_{\gamma \in-2 \mathcal{Q}^{+} \backslash\{0\}} R_{0}^{w}[\gamma] \subseteq Q(w)_{w}^{+}$. Assume that $R_{0}^{w}[0] \cap Q(w)_{w}^{+} \neq 0$. Then there exist $\lambda \in \mathcal{P}^{+}$and a homogeneous element $\xi \in V(\lambda)^{*}, \xi \neq 0$ such that $c_{\xi}^{\lambda} e_{w}^{-\lambda} \in R_{0}^{w}[0]$ and $\xi\left(\mathcal{U}^{+} T_{w} v_{\lambda}\right)=0$. Because of (3.10), wt $\left(\left(\partial_{w^{-1}}\left(c_{\xi}^{\lambda}\right)\right) e_{1}^{-\lambda}\right)=0$, so $\partial_{w^{-1}}\left(c_{\xi}^{\lambda}\right)=s e_{1}^{\lambda}$ for some $s \in$ $\mathbb{K}^{*}$. Thus, $\xi\left(\left(X_{i_{1}}^{-}\right)^{m_{1}} \ldots\left(X_{i_{l}}^{-}\right)^{m_{l}} v_{\lambda}\right) \neq 0$ for some $m_{1}, \ldots, m_{l} \in \mathbb{N}$, where $i_{1}, \ldots, i_{l}$ are the indices of the reduced decomposition (2.11) of $w$. Yet, $\left(X_{i_{1}}^{-}\right)^{m_{1}} \ldots\left(X_{i_{l}}^{-}\right)^{m_{l}} v_{\lambda} \in \mathcal{U}^{+} T_{w} v_{\lambda}$ by [27, Lemma 4.4.3(v)], so $\xi\left(\left(X_{i_{1}}^{-}\right)^{m_{1}} \ldots\left(X_{i_{l}}^{-}\right)^{m_{l}} v_{\lambda}\right)=0$ because $\xi\left(\mathcal{U}_{+} T_{w} v_{\lambda}\right)=0$. Thus, the assumption is not correct and

$$
Q(w)_{w}^{+}=\oplus_{\gamma \in-2 \mathcal{Q}^{+} \backslash\{0\}} R_{0}^{w}[\gamma] .
$$

Moreover, $R_{0}^{w}[0]$ is a subalgebra of $R_{0}^{w}$. The ideal $Q(y)_{w}^{-}$is $\psi_{w}^{\mu}$-stable and homogeneous with respect to the grading (2.16), thus $Q(y)_{w}^{-}=\left(Q(y)_{w}^{-} \cap R_{0}^{w}[0]\right) \oplus$ $\left(Q(y)_{w}^{-} \cap\left(\oplus_{\gamma \in-2 \mathcal{Q}^{+} \backslash\{0\}} R_{0}^{w}[\gamma]\right)\right)$ and

$$
\begin{aligned}
R_{0}^{w} /\left(Q(y)_{w}^{-}+Q(w)_{w}^{+}\right) & \cong R_{0}^{w}[0] /\left(Q(y)_{w}^{-} \cap R_{0}^{w}[0]\right) \\
& \cong\left(Q(y)_{w}^{-}+R_{0}^{w}[0]\right) / Q(y)_{w}^{-} \hookrightarrow R_{0}^{w} / Q(y)_{w}^{-} .
\end{aligned}
$$

Therefore, $Q(y)_{w}^{-}+Q(w)_{w}^{+}$is a completely prime ideal of $R_{0}^{w}$, since $R_{0}^{w} / Q(y)_{w}^{-}$is a domain by Lemma 3.4.

Next, we go over the key steps of Gorelik's proof of (3.7) to show that it works for all base fields $\mathbb{K}, q \in \mathbb{K}^{*}$ not a root of unity. We have that $e_{y}^{\lambda} e_{w}^{-\lambda} \in Q(w)_{w}^{+}+Q(y)_{w}^{-}$ if and only if $e_{y}^{\lambda} e_{w}^{\lambda^{\prime}} \in Q(w)^{+}+Q(y)^{-}$for some $\lambda^{\prime} \in \mathcal{P}^{+}$. (For some of the arguments below, $\lambda^{\prime}$ should be chosen sufficiently large.) Define $s_{i} \star w=\max \left(s_{i} w, w\right)$. Let $s_{i} y>y$ and $y \leq w$. Then $s_{i} y \leq s_{i} \star w,[27$, Proposition A.1.7]. Gorelik proves that

$$
e_{y}^{\lambda} e_{w}^{\lambda^{\prime}} \in Q(w)^{+}+Q(y)^{-} \Rightarrow e_{s_{i} y}^{\lambda} e_{s_{i} \star w}^{\lambda^{\prime}} \in Q\left(s_{i} \star w\right)^{+}+Q\left(s_{i} y\right)^{-}
$$

as described below. Since (3.7) is obvious for $y=w=w_{0}$, (3.7) follows from (3.13) by induction.

Define the automorphisms $\bar{\sigma}_{i}=\left(\left\llcorner K_{i}\right)\right.$ of $R^{+}$and the (left skew) derivations $\bar{\partial}_{i}=\left(\left\llcorner X_{i}^{+}\right), i \in[1, r]\right.$, cf. Section 2. For a homogeneous element $r \in R^{+}$with respect 
to the grading (2.15) in an analogy to (3.9), define $\bar{\partial}_{i}^{*}(r)=\bar{\partial}_{i}^{n}(r)$, where $n=\operatorname{deg}_{\bar{\partial}_{i}} r$. Since $\mathbb{K}\left[X_{i}^{+}\right] \mathcal{U}_{-} T_{s_{i}} v_{\lambda} \subseteq \mathcal{U}_{-} T_{y} v_{\lambda}, \forall \lambda \in \mathcal{P}^{+}$,

$$
\bar{\partial}_{i}^{n} Q(y)^{-} \subseteq Q\left(s_{i} y\right)^{-}, \quad \forall n \in \mathbb{N} .
$$

If $s_{i} w<w$, then $\operatorname{deg}_{\bar{\partial}_{i}} e_{w}^{\lambda^{\prime}}=0$ and $e_{s_{i} y}^{\lambda} e_{w}^{\lambda^{\prime}}=t \bar{\partial}_{i}^{*}\left(e_{y}^{\lambda} e_{w}^{\lambda^{\prime}}\right) \in Q(w)^{+}+Q\left(s_{i} y\right)^{-}$for some $t \in$ $\mathbb{K}^{*}$, because $Q(w)^{+}$is $\bar{\partial}_{i}$-invariant. This proves (3.13) in the case $s_{i} w<w$. The case $s_{i} w>w$ of (3.13) is more complicated. First one decomposes according to (3.11):

$$
\begin{aligned}
e_{y}^{\lambda} e_{w}^{-\lambda} & =b_{0}+\ldots+b_{m} \text { for } b_{0} \in Q(y)_{w}^{-} \cap R_{0}^{w}[0], b_{j} \\
& \in R_{0}^{w}\left[-2 \gamma_{j}\right], \gamma_{j} \in \mathcal{Q}^{+} \backslash\{0\}, j \in[1, m],
\end{aligned}
$$

$\gamma_{j} \neq \gamma_{j^{\prime}}$ for $j \neq j^{\prime}$. Then

$$
\operatorname{wt}\left(b_{j}\right)=\operatorname{wt}\left(e_{y}^{\lambda} e_{w}^{-\lambda}\right)=(w-y) \lambda, \quad \forall j \in[0, m] .
$$

Denote $n:=\operatorname{deg}_{\bar{a}_{i}^{*}}\left(e_{y}^{\lambda} e_{w}^{\lambda^{\prime}}\right)$. Since the elements $b_{0}, \ldots, b_{m}$ are linearly independent, cf. (3.11),

$$
\begin{aligned}
& \operatorname{deg}_{\bar{\partial}_{i}^{*}}\left(b_{j} e_{w}^{\lambda+\lambda^{\prime}}\right) \leq n, \quad \forall j \in[0, m] \quad \text { and } \\
& e_{s_{i}}^{\lambda} y_{s_{i} w}^{\lambda^{\prime} w}=t \bar{\partial}_{i}^{*}\left(e_{y}^{\lambda} e_{w}^{\lambda^{\prime}}\right)=\sum_{j \in M} t \bar{\partial}_{i}^{*}\left(b_{j} e_{w}^{\lambda+\lambda^{\prime}}\right), t \in \mathbb{K}^{*},
\end{aligned}
$$

where $M=\left\{j \in[0, m] \mid \operatorname{deg}_{\bar{\partial}_{i}^{*}}\left(b_{j} e_{w}^{\lambda+\lambda^{\prime}}\right)=n\right\}$. Analogously to (3.8) one shows that for $s_{i} w>w$, if $r \in R^{+}$is homogeneous and $\mathrm{wt}_{w}(r)$ exists, then $\mathrm{wt}_{s_{i} w}\left(\bar{\partial}_{i} r\right)$ exists and

$$
\mathrm{wt}_{w}(r)+w^{-1} \operatorname{wt}(r)=\mathrm{wt}_{s_{i} w}\left(\bar{\partial}_{i} r\right)+\left(s_{i} w\right)^{-1} \operatorname{wt}\left(\bar{\partial}_{i} r\right) .
$$

Equations (3.15) and (3.16) imply that $\operatorname{wt}\left(\bar{\partial}_{i}^{*}\left(b_{j} e_{w}^{\lambda+\lambda^{\prime}}\right)\right)=\operatorname{wt}\left(e_{s_{i} y}^{\lambda} y_{s_{i} w}^{\lambda^{\prime}}\right)$ for all $j \in M$. It follows from this, (3.17) and (3.12) that $\sum_{j \in M \backslash\{0\}} \bar{\partial}_{i}^{*}\left(b_{j} e_{w}^{\lambda+\lambda^{\prime}}\right) \in Q\left(s_{i} w\right)^{+}$. Equation (3.14) implies that $\bar{\partial}_{i}^{*}\left(b_{0} e_{w}^{\lambda+\lambda^{\prime}}\right) \in Q\left(s_{i} y\right)^{-}$, which completes the proof of (3.13) in the case $s_{i} w>w$.

3.3. Strata of prime ideals. For $J \in \operatorname{Spec} \mathcal{U}_{-, \mathbf{p}}^{w}$ and $\lambda \in \mathcal{P}^{+}$denote

$$
C_{J}(\lambda)=\left\{v \in \mathcal{P} \mid \exists \xi \in\left(V(\lambda)^{*}\right)_{v}, b_{w, \xi}^{\lambda} \notin J\right\} .
$$

For all $\lambda \in \mathcal{P}^{+}$, we have $-w \lambda \in C_{J}(\lambda)$ since $a_{w, w}^{\lambda}=1 \notin J$. Denote by $D_{J}(\lambda)$ the set of minimal elements of the set $C_{J}(\lambda)$.

Lemma 3.6. For all base fields $\mathbb{K}, q \in \mathbb{K}^{*}$ not a root of unity, $w \in W, \mathbf{p} \in$ $Z^{2}\left(\mathcal{Q}_{\mathcal{S}(w)}, \mathbb{K}^{*}\right)_{n}$ and $I \in \mathrm{Spec} \mathcal{U}_{-, \mathrm{p}}^{w}$, there exists a unique $y \in W^{\leq w}$ such that $D_{J}(\lambda)=$ $\{-y \lambda\}$ for all $\lambda \in \mathcal{P}^{+}$.

Gorelik's analogous result [20, Section 5.2.1] was formulated under the assumption $\mathbb{K}=k(q)$ for a field $k$, char $k=0$. This proof works under the more general assumptions on $\mathbb{K}$ and $q$. We sketch this below. 
Proof of Lemma 3.6. Fix $J \in \operatorname{Spec}_{-, \mathbf{p}}^{w}$. Let $\lambda \in \mathcal{P}^{+}, v \in D_{J}(\lambda), \xi \in\left(V(\lambda)^{*}\right)_{v}, b_{w, \xi}^{\lambda} \notin$ $J$. Applying (2.26), we obtain that $b_{w, \xi}^{\lambda}$ defines a non-zero normal element of $\mathcal{U}_{-, \mathbf{p}}^{w} / J$ :

$$
b_{w, \xi}^{\lambda} x=\mathbf{r}(v+w \lambda, \gamma) q^{\langle\nu-w \lambda, \gamma\rangle} x b_{w, \xi}^{\lambda} \quad \bmod J, \quad \forall x \in\left(\mathcal{U}_{-, \mathbf{p}}^{w}\right)_{\gamma}, \gamma \in \mathcal{Q}_{\mathcal{S}(w)} .
$$

Applying this twice and using that $\mathbf{r}(.$, .) is multiplicatively skew symmetric (see Section 2), we obtain that for all $\lambda_{i} \in \mathcal{P}^{+}, v_{i} \in D_{J}\left(\lambda_{i}\right), \xi_{i} \in\left(V\left(\lambda_{i}\right)^{*}\right)_{v_{i}}, b_{w, \xi_{i}}^{\lambda_{i}} \notin J, i=1,2$,

$$
b_{w, \xi_{1}}^{\lambda_{1}} b_{w, \xi_{2}}^{\lambda_{2}}=q^{\left\langle\nu_{1}-w \lambda_{1}, \nu_{2}+w \lambda_{2}\right\rangle+\left\langle\nu_{2}-w \lambda_{2}, \nu_{1}+w \lambda_{1}\right\rangle} b_{w, \xi_{1}}^{\lambda_{1}} b_{w, \xi_{2}}^{\lambda_{2}} \bmod J
$$

Since $J$ is prime and the images of $b_{w, \xi_{i}}^{\lambda_{i}}$ in $\mathcal{U}_{-, \mathbf{p}}^{w} / J$ are non-zero normal elements, they are regular. Using the fact that $q \in \mathbb{K}^{*}$ is not a root of unity, we obtain

$$
\left\langle v_{1}-w \lambda_{1}, v_{2}+w \lambda_{2}\right\rangle+\left\langle v_{2}-w \lambda_{2}, v_{1}+w \lambda_{1}\right\rangle=0, \text { so }\left\langle v_{1}, v_{2}\right\rangle=\left\langle\lambda_{1}, \lambda_{2}\right\rangle .
$$

By [27, Lemma A.1.17], if $\lambda_{1}, \lambda_{2} \in \mathcal{P}^{++}$, then $v_{i}=-y \lambda_{i}, i=1,2$, for some $y \in W$. Therefore, there exists $y \in W$ such that $D_{J}(\lambda)=\{-y \lambda\}, \forall \lambda \in \mathcal{P}^{++}$. Let $\lambda \in \mathcal{P}^{++}$. Since $b_{w, \xi}^{\lambda} \neq 0$ for some $\xi \in\left(V(\lambda)^{*}\right)_{-y \lambda}$ and $\operatorname{ker} \phi_{w}^{+}=Q(w)_{w}^{+}$(see Proposition 2.2), we have $\left(\mathcal{U}_{+} T_{w} v_{\lambda}\right)_{y \lambda} \neq 0$. By [27, Proposition 4.4.5], $y \leq w$. Analogous to the proof of [27, Proposition 9.3.8] one shows that $D_{J}(\lambda)=\{-y \lambda\}, \forall \lambda \in \mathcal{P}^{+}$for the same Weyl group element $y$.

3.4. Proof of the main result. We proceed with the proof of Theorem 3.1. For $y \in W^{\leq w}$ denote

$$
\operatorname{Spec}_{y} \mathcal{U}_{-, \mathbf{p}}^{w}=\left\{J \in \operatorname{Spec}_{-, \mathbf{p}}^{w} \mid D_{J}(\lambda)=\{-y \lambda\}, \forall \lambda \in \mathcal{P}^{+}\right\} .
$$

The definition of the sets $D_{J}(\lambda)$ and Lemma 3.6 imply

$$
\operatorname{Spec}_{-, \mathbf{p}}^{w}=\bigsqcup_{y \in W \leq w} \operatorname{Spec}_{y} \mathcal{U}_{-, \mathbf{p}}^{w}
$$

Proof of Theorem 3.1. By Lemma 3.5(b), $I_{w}(y) \in \operatorname{Spec}_{y} \mathcal{U}_{-, \mathbf{p}}^{w}, \forall y \in W^{\leq w}$. Since $\operatorname{dim}(V(\lambda))_{y \lambda}=1, \forall \lambda \in \mathcal{P}^{+}, y \in W$, each stratum $\operatorname{Spec}_{y} \mathcal{U}_{-, \mathbf{p}}^{w}$ contains a unique $\mathbb{T}^{|\mathcal{S}(w)|_{-}}$ invariant prime ideal, which would have to be precisely the ideal $I_{w}(y)$. This implies part (a) of the theorem except the statement for the poset structure of $\mathbb{T}^{|\mathcal{S}(w)|}-\operatorname{Spec}_{-, \mathbf{p}}^{w}$. It also implies

$$
I_{w}(y)=\cap_{t \in \mathbb{T}|\mathcal{S}(w)|} t . J, \quad \forall J \in \operatorname{Spec}_{y} \mathcal{U}_{-, \mathbf{p}}^{w},
$$

recall (2.23). Furthermore, we have

$$
\operatorname{Spec}_{y} \mathcal{U}_{-, \mathbf{p}}^{w}=\left\{J \in \operatorname{Spec}_{-, \mathbf{p}}^{w} \mid J \supseteq I_{w}(y) \text { and } J \cap A_{y, w}=\emptyset\right\} .
$$

The left inclusion in (3.20) follows from (3.19) and the definition of the sets $D_{J}(\lambda)$. The equality follows from (3.18).

To complete the proof of part (a), note that $y_{1} \leq y_{2} \in W^{\leq w}$ implies $\mathcal{U}_{-} T_{y_{1}} v_{\lambda} \supseteq$ $\mathcal{U}_{-} T_{y_{2}} v_{\lambda}$, see [27, Lemma 4.4.3], therefore $Q\left(y_{1}\right)_{w}^{-} \subseteq Q\left(y_{2}\right)_{w}^{-}$and $I_{w}\left(y_{1}\right)=\phi_{w}^{+}\left(Q\left(y_{1}\right)_{w}^{-}\right) \subseteq$ $\phi_{w}^{+}\left(Q\left(y_{2}\right)_{w}^{-}\right)=I_{w}\left(y_{2}\right)$. Now assume that $I_{w}\left(y_{1}\right) \subseteq I_{w}\left(y_{2}\right)$ for some $y_{1}, y_{2} \in W^{\leq w}$. Since $a_{y_{2}, w}^{\lambda} \notin I_{w}\left(y_{2}\right)$ by Lemma 3.5, $a_{y_{2}, w}^{\lambda} \notin I_{w}\left(y_{1}\right), \forall \lambda \in \mathcal{P}^{+}$. Therefore, $T_{y_{2}} v_{\lambda} \in \mathcal{U}_{-} T_{y_{1}} v_{\lambda}$ (because otherwise we would get $c_{y_{2}}^{\lambda} e_{w}^{-\lambda} \in Q\left(y_{1}\right)_{w}^{-}$and $a_{y_{2}, w}^{\lambda} \in I_{w}\left(y_{1}\right)$ ). Applying this to any $\lambda \in \mathcal{P}^{++}$gives $y_{1} \leq y_{2}$ by [27, Proposition 4.4.5]. 
Part (c) follows from (3.18) and (3.20). The rings $R_{y, w}$ are $\mathbb{T}^{|\mathcal{S}(w)|}$-simple, since otherwise a $\mathbb{T}^{|\mathcal{S}(w)|}$-invariant maximal ideal of $R_{y, w}$ will contract to a $\mathbb{T}^{|\mathcal{S}(w)|}$-invariant prime ideal in the stratum $\operatorname{Spec}_{y} \mathcal{U}_{-, \text {p }}^{w}$, which properly contains $I_{w}(y)$. This will contradict with the fact that $I_{w}(y)$ is the only $\mathbb{T}^{|\mathcal{S}(w)|}$-invariant prime ideal in $\operatorname{Spec}_{y} \mathcal{U}_{-, \mathbf{p}}^{w}$. This and Lemma 3.5(b) prove part (b) of the theorem.

It follows from Lemma 2.4 that Goodearl's result [4, Theorem II.6.4] is applicable to the algebras $\mathcal{U}_{-, \mathbf{p}}^{w}$ and as a consequence of this all of their $\mathbb{T}^{|\mathcal{S}(w)|}$-prime ideals are strongly rational. This means that the zero components of the centres $R_{y, w}$ with respect to the grading (2.3) reduce to scalars: $\left(Z\left(R_{y, w}\right)\right)_{0}=\mathbb{K}, \forall y \leq w$. Next, we apply two results of Goodearl and Letzter: [18, Lemma 6.3 (c)] implies that

$$
\operatorname{dim}\left(Z\left(R_{y, w}\right)\right)_{\gamma}=0 \text { or } 1, \text { for all } \gamma \in \mathcal{Q}_{\mathcal{S}(w)}
$$

and that $Z\left(R_{y, w}\right)$ are Laurent polynomial rings over $\mathbb{K}$. Furthermore, [18, Corollary 6.5] gives that contraction and extension provide mutually inverse homeomorphisms between $\operatorname{Spec} Z\left(R_{y, w}\right)$ and $\operatorname{Spec} R_{y, w}$, for all $y \leq w$. Finally, because of (3.20) and general localization facts $J \mapsto\left(J / I_{w}(y)\right) R_{y, w}$ is a homeomorphism between $\operatorname{Spec}_{y} \mathcal{U}_{-, \mathbf{p}}^{w}$ and $\operatorname{Spec} R_{y, w}$. Equation (3.3) follows from part (a) and (3.19). This completes the proof of part (d).

\section{Dimensions of the Goodearl-Letzter strata of $\mathcal{U}_{-, p}^{w}$.}

4.1. The untwisted case. The spectra of the algebras $\mathcal{U}_{-, \mathrm{p}}^{w}$ are partitioned (3.2) into disjoint unions of the Goodearl-Letzter strata $\operatorname{Spec}_{I_{w}(y)} \mathcal{U}_{-, \mathbf{p}}^{w}$, cf. (3.4). Each of them is homeomorphic to the spectrum of a Laurent polynomial ring over $\mathbb{K}$, namely $Z\left(R_{y, w}\right)$ (see Theorem $3.1(\mathrm{~d})$ ). To determine Spec $\mathcal{U}_{-, \mathbf{p}}^{w}$ set theoretically, one needs to solve the problem for computing the dimensions of these Laurent polynomial rings. In this section we obtain an explicit formula for these dimensions. This is done in Theorem 4.5, and Sections 5-5 contain some preparatory results. We note that unlike Theorem 3.1, the twisted case of Theorem 4.5 is more difficult than the untwisted case and cannot be deduced from the latter.

Let $\mu \in \mathcal{P}$. If $\mu=\lambda_{1}-\lambda_{2}$ for some $\lambda_{1}, \lambda_{2} \in \mathcal{P}^{+}$with disjoint support, define

$$
a_{y, w}^{\mu}=a_{y, w}^{\lambda_{1}}\left(a_{y, w}^{\lambda_{2}}\right)^{-1} \in R_{y, w} .
$$

Since $\mathbf{r}(.$, . ) is a bi-character, see (2.20), equation (3.1) implies that

$$
a_{y, w}^{\mu} x=\mathbf{r}((w-y) \mu, \gamma) q^{-\langle(w+y) \mu, \gamma\rangle} x a_{y, w}^{\mu}, \quad \forall \mu \in \mathcal{P}, \gamma \in \mathcal{Q}_{\mathcal{S}(w)}, x \in\left(R_{y, w}\right)_{\gamma} .
$$

Recall (3.21). For $y \in W^{\leq w}$ denote the sublattice of $\mathcal{Q}_{\mathcal{S}(w)}$

$$
\mathcal{Z}_{y, w}=\left\{\gamma \in \mathcal{Q}_{\mathcal{S}(w)} \mid\left(Z\left(R_{y, w}\right)\right)_{\gamma} \neq 0\right\} .
$$

By Theorem 3.1(d),

$$
Z\left(R_{y, w}\right) \text { is a Laurent polynomial ring over } \mathbb{K} \text { of dimension equal to } \operatorname{rank} \mathcal{Z}_{y, w} \text {. }
$$

The special case for untwisted quantum Schubert cell algebras of the general dimension result in the below-stated Theorem 4.5 was previously obtained by Bell 
et al. in [3] and Yakimov in [39] under the additional condition that char $\mathbb{K}=0$ and $q$ is transcendental over $\mathbb{Q}$. We give a very simple new derivation of this formula.

Proposition 4.1. ([3, 39]). For all base fields $\mathbb{K}, q \in \mathbb{K}^{*}$ not a root of unity, and Weyl group elements $y \leq w$, the Goodearl-Letzter strata $\operatorname{Spec}_{I_{w}(y)} \mathcal{U}_{-}^{w}$ are homeomorphic to the spectra Laurent polynomial rings over $\mathbb{K}$ of dimension equal to $\operatorname{dim} \operatorname{Ker}(w+y)$.

Proof. If $\mu \in \operatorname{Ker}_{\mathcal{P}}(w+y)=\{\mu \in \mathcal{P} \mid(w+y) \mu=0\}$, then by (4.2), $a_{y, w}^{\mu} \in$ $\left(Z\left(R_{y, w}\right)\right)_{(w-y) \mu} \backslash\{0\}$. Therefore, $\mathcal{Z}_{y, w} \supseteq(w-y) \operatorname{Ker}_{\mathcal{P}}(w+y)=2 w \operatorname{Ker}_{\mathcal{P}}(w+y)$. So $\operatorname{rank} \mathcal{Z}_{y, w} \geq \operatorname{dim} \operatorname{ker}(w+y)$.

Let $z \in\left(Z\left(R_{y, w}\right)\right)_{\gamma} \backslash\{0\}, \gamma \in \mathcal{Q}_{\mathcal{S}(w)}$. The central property of $z$ and (4.2) imply

$$
a_{y, w}^{\mu} z=q^{-\langle(w+y) \mu, \gamma\rangle} z a_{y, w}^{\mu}=q^{-\langle(w+y) \mu, \gamma\rangle} a_{y, w}^{\mu} z
$$

for all $\mu \in \mathcal{P}$. Since $q \in \mathbb{K}^{*}$ is not a root of unity, $\gamma \in((w+y) \mathcal{P})^{\perp} \cap$ $\mathcal{Q}$, i.e. $\quad \mathcal{Z}_{y, w} \subseteq((w+y) \mathcal{P})^{\perp} \cap \mathcal{Q}$. Because $\operatorname{rank}((w+y) \mathcal{P})^{\perp} \cap \mathcal{Q}=\operatorname{dim} \operatorname{ker}(w+$ $y), \quad \operatorname{rank} \mathcal{Z}_{y, w} \leq \operatorname{dim} \operatorname{ker}(w+y)$. Therefore, $\operatorname{rank} \mathcal{Z}_{y, w}=\operatorname{dim} \operatorname{ker}(w+y)$, and the proposition follows from Theorem 3.1(d), cf. (4.4).

4.2. Supports of prime factors. Fix a reduced expression

$$
s_{i_{1}} \ldots s_{i_{l}}
$$

of $w \in W$ and recall (2.12). We will identify the subexpressions of (4.5) with the subsets $D \subseteq[1, l]$. For $D \subset[1, l]$ set $s_{i_{j}}^{D}=s_{i_{j}}$ if $j \in D$ and $s_{i_{j}}^{D}=1$ otherwise. Define $w_{(j)}=$ $s_{i_{1}} \ldots s_{i_{j}}, w_{(j)}^{D}=s_{i_{1}}^{D} \ldots s_{i_{j}}^{D}, w^{D}=w_{(l)}^{D}$ and $\bar{w}_{(j)}^{D}=s_{i_{j+1}}^{D} \ldots s_{i_{l}}^{D}, j \in[1, l]$. A subexpression $D \subseteq[1, l]$ is called a Cauchon diagram if for all $j \in[1, l-1], s_{i j} \bar{w}_{(j)}^{D}>\bar{w}_{(j)}^{D}$. Taking inverses establishes a bijection between the set of those and the set of the positive subexpressions of Marsh and Rietsch [32] of the reverse expression of (4.5). By [32, Lemma 3.5] for each $y \in W^{\leq w}$, there exists a unique Cauchon diagram $D \subseteq[1, l]$ such that $w^{D}=y$. Using it, define the lattice

$$
\mathcal{Q}_{y, w}=\sum_{j \in[1, l] \backslash D} \mathbb{Z} w_{(j-1)}^{D}\left(\alpha_{j}\right) .
$$

We will see shortly that this does not depend on the choice of a reduced expression of $w$. The following lemma provides a second characterization of $\mathcal{Q}_{y, w}$.

Lemma 4.2. Let $y \leq w \in W$. For a reduced expression (4.5) of $w$, let $D \subseteq[1, l]$ be the Cauchon diagram such that $w^{D}=y$. In the notation from (2.12):

$$
\mathcal{Q}_{y, w}=\sum_{j \in[1,] \backslash D} \mathbb{Z} \beta_{i_{j}} .
$$

By [39, Lemma 3.2(ii)], $\mathcal{Q}_{\mathcal{S}(w)}=\mathbb{Z} \beta_{1}+\ldots+\mathbb{Z} \beta_{l}$. Thus, $\mathcal{Q}_{y, w}$ is a sub-lattice of $\mathcal{Q}_{\mathcal{S}(w)}$. Lemma 4.2 follows at once form the fact that

$$
s_{i_{1}} \ldots s_{i_{j}}\left(\alpha_{i_{j+1}}\right)=s_{i_{1}} \ldots s_{i_{j-1}}\left(\alpha_{i_{j+1}}\right) \quad \bmod \left(\mathbb{Z} s_{i_{1}} \ldots s_{i_{j-1}}\left(\alpha_{i_{j}}\right)\right), \quad \forall j \in[2, l-1] .
$$


For $y \in W^{\leq w}$ denote by $\operatorname{Supp}\left(\mathcal{U}_{-, \mathbf{p}}^{w} / I_{w}(y)\right)$ the subgroup of $\mathcal{Q}_{\mathcal{S}(w)}$ generated by $\gamma \in$ $\mathcal{Q}_{\mathcal{S}(w)}^{+}$such that $\left(\mathcal{U}_{-, \mathbf{p}}^{w} / I_{w}(y)\right)_{\gamma} \neq 0$, which is the same as the subgroup generated by $\gamma \in \mathcal{Q}_{\mathcal{S}(w)}$ such that $\left(R_{y, w}\right)_{\gamma} \neq 0$. Obviously, these supports do not depend on $\mathbf{p}$. The next result describes them. (As a side result it also implies that $\mathcal{Q}_{y, w}$ is independent of the choice of a reduced expression of $w$.)

THEOREM 4.3. For all base fields $\mathbb{K}, q \in \mathbb{K}^{*}$ not a root of unity, Weyl group elements $y \leq w$ and 2-cocycles $\mathbf{p} \in Z^{2}\left(\mathcal{Q}_{\mathcal{S}(w)}, \mathbb{K}^{*}\right)_{n}$,

$$
\operatorname{Supp}\left(\mathcal{U}_{-, \mathbf{p}}^{w} / I_{w}(y)\right)=\mathcal{Q}_{y, w} .
$$

Proof. We will first show that

$$
\operatorname{Supp}\left(\mathcal{U}_{-}^{w} / I_{w}(y)\right) \supseteq \mathcal{Q}_{y, w} .
$$

We will use the fact that if $y^{\prime}, y^{\prime \prime} \in W$ and $i \in[1, r]$ are such that $y^{\prime} s_{i}>y^{\prime}$ and $s_{i} y^{\prime \prime}>y^{\prime \prime}$, then $y^{\prime} s_{i} y^{\prime \prime}>y^{\prime} y^{\prime \prime}$. In terms of the reduced expression (4.5), let $D \subseteq[1, l]$ be the Cauchon diagram such that $y=w^{D}$. Denote $[1, l] \backslash D=\left\{j_{1}<\ldots<j_{l-|D|}\right\}$. For $m \in[0, l-|D|]$, define

$$
D_{m}=D \sqcup\left\{j_{1}, \ldots j_{m}\right\}=D \cup\left[1, j_{m}\right], y_{m}=w^{D_{m}} .
$$

Then $y_{0}=y, y_{l-|D|}=w$ and $y_{m}=w_{\left(j_{m}\right)} \bar{w}_{\left(j_{m}\right)}^{D}=w_{\left(j_{m}-1\right)} s_{i_{m}} \bar{w}_{\left(j_{m}\right)}^{D}, \forall m=1, \ldots, l-|D|$. Moreover, $y_{m-1}=w_{\left(j_{m}-1\right)} \bar{w}_{\left(j_{m}\right)}^{D}, \forall m=1, \ldots, l-|D|$. Since (4.5) is a reduced expression $w_{\left(j_{m}-1\right)} s_{i_{j m}}>w_{\left(j_{m}-1\right)}$. Because $D$ is a Cauchon diagram, $s_{i_{j_{m}}} \bar{w}_{\left(j_{m}\right)}^{D}>\bar{w}_{\left(j_{m}\right)}^{D}$. By the above-mentioned fact, $y=y_{0}<y_{1}<\ldots<y_{l-|D|}=w$. By Theorem 3.1(a)-(b), $a_{y_{m}, w}^{\lambda} \notin I_{w}(y), \forall m \in[1, l-|D|]$. Therefore, $a_{y_{m}, w}^{\lambda} \in\left(\mathcal{U}_{-}^{w} / I_{w}(y)\right)_{\left(w-y_{m}\right) \lambda}$ and $a_{y_{m-1}, w}^{\lambda} \in$ $\left(\mathcal{U}_{-}^{w} / I_{w}(y)\right)_{\left(w-y_{m-1}\right) \lambda}$. By an easy computation one obtains that $y_{m} \lambda=y_{m-1} \lambda-$ $\left\langle\bar{w}_{\left(j_{m}\right)}^{D} \lambda, \alpha_{i_{j_{m}}}^{\vee}\right\rangle \beta_{j_{m}}$ in terms of notation (2.12). Thus,

$$
\left\langle\bar{w}_{\left(j_{m}\right)}^{D} \lambda, \alpha_{i_{j_{m}}}^{\vee}\right\rangle \beta_{j_{m}} \in \operatorname{Supp}\left(\mathcal{U}_{-}^{w} / I_{w}(y)\right), \quad \forall \lambda \in \mathcal{P}^{+}, m \in[1, l-|D|] .
$$

Since $\operatorname{gcd}\left\{\left\langle\vec{a}_{\left(j_{m}\right)}^{D} \lambda, \alpha_{i_{j_{m}}}^{\vee}\right\rangle \mid \lambda \in \mathcal{P}^{+}\right\}=1$,

$$
\beta_{j} \in \operatorname{Supp}\left(\mathcal{U}_{-}^{w} / I_{w}(y)\right), \quad j \in[1, l] \backslash D .
$$

Now (4.7) follows from Lemma 4.2.

Mériaux and Cauchon [33] gave another classification of $\mathbb{T}^{|\mathcal{S}(w)|}{ }_{-}$Spec $\mathcal{U}_{-}^{w}$, associating to each $y \in W^{\leq w}$, an ideal $J_{w}(y) \in \mathbb{T}^{|\mathcal{S}(w)|}-\mathrm{Spec} \mathcal{U}_{-}^{w}$. It is based on the Cauchon method of deleting derivations [7], which is very different from the one given in [38]. It is not known yet whether $J_{w}(y)=I_{w}(y), \forall y \in W \leq w$. Mériaux and Cauchon [33] proved that a certain localization of $\mathcal{U}_{-}^{w} / J_{w}(y)$ by an Ore subset of homogeneous elements is isomorphic to a quantum torus with generators of weight $\left\{\beta_{i_{n}} \mid n \in[1, l] \backslash D\right\}$, where $D$ is the Cauchon diagram such that $y=w^{D}$. Combining this with Lemma 4.2 implies that $\operatorname{Supp}\left(\mathcal{U}_{-}^{w} / J_{w}(y)\right)=\mathcal{Q}_{y, w}, \forall y \in W \leq w$. Since $\left\{I_{w}(y) \mid y \in\right.$ $\left.W^{\leq w}\right\}=\left\{J_{w}(y) \mid y \in W^{\leq w}\right\}$ and $\operatorname{Supp}\left(\mathcal{U}_{-}^{w} / I_{w}(y)\right) \supseteq \mathcal{Q}_{y, w}, \forall y \in W^{\leq w}$ we have

$$
\operatorname{Supp}\left(\mathcal{U}_{-}^{w} / I_{w}(y)\right)=\mathcal{Q}_{y, w}, \quad \forall y \in W^{\leq w} .
$$


4.3. Normal elements. Assume that $R$ is a $\mathbb{K}$-algebra graded by a group $C$. We will say that $u \in R$ is a diagonal normal element if $u \in R_{\gamma_{0}}$ for some $\gamma_{0} \in C$, and for all $\gamma \in C$ there exists $t_{\gamma} \in \mathbb{K}^{*}$ such that $u r=t_{\gamma} r u, \forall r \in R_{\gamma}$. If $R$ is a prime ring and $u \neq 0$, then $t: C \rightarrow \mathbb{K}^{*}$ is a character.

For all $y \leq w \in W$ there exists $n_{y, w} \in \mathbb{Z}_{+}$such that all homomorphisms $\mathcal{Q}_{y, w} \rightarrow \mathbb{Z}$ have the form $\gamma \in \mathcal{Q}_{y, w} \mapsto\langle\lambda, \gamma\rangle$ for some $\lambda \in\left(1 / n_{y, w}\right) \mathcal{P}$.

Proposition 4.4. For all base fields $\mathbb{K}, q \in \mathbb{K}^{*}$ not a root of unity, Weyl group elements $y \leq w$, 2-cocycles $\mathbf{p} \in Z^{2}\left(\mathcal{Q}_{\mathcal{S}(w)}, \mathbb{K}^{*}\right)_{n}$ and non-zero diagonal normal elements $u \in R_{y, w}$, there exits $\mu \in\left(1 / 2 n_{y, w}\right) \mathcal{P}$ such that $(w-y) \mu \in \mathcal{Q}_{\mathcal{S}(w)}, u \in\left(R_{y, w}\right)_{(w-y) \mu}$, $\left\langle(w+y) \mu, \mathcal{Q}_{y, w}\right\rangle \subseteq \mathbb{Z}$ and

$$
u x=\mathbf{r}((w-y) \mu, \gamma) q^{-\langle(w+y) \mu, \gamma\rangle} x u, \quad \forall \gamma \in \mathcal{Q}_{y, w}, x \in\left(R_{y, w}\right)_{\gamma}
$$

in terms of the integers $n_{y, w} \in \mathbb{Z}_{+}$defined above.

Proof. The set of diagonal normal elements of an algebra graded by an abelian group is invariant under twisting. Because of this, one only needs to prove the proposition for the algebras $\mathcal{U}_{-}^{w}$, i.e. when $\mathbf{p}$ is trivial.

Let $u \in \mathcal{U}_{-}^{w} / I_{w}(y)$ be such that $u\left(a_{y, w}^{\lambda}\right)^{-1} \in R_{y, w}$ is a non-zero diagonal normal element for some $\lambda \in \mathcal{P}^{+}$. Then (4.2) implies that $u$ is a non-zero diagonal normal element of $\mathcal{U}_{-}^{w} / I_{w}(y)$. By the same reasoning, if we establish the proposition for $u$, then its validity for $u\left(a_{y, w}^{\lambda}\right)^{-1}$ will follow. Recall (4.5) and (2.12). By Cauchon's method of deleting derivations [7] and the nature of the iterated Ore extension $\mathcal{U}_{-}^{w}$ given by Lemma 2.4 (see [33]), it follows that there exists a localization of $\mathcal{U}_{-}^{w} / I_{w}(y)$ by an Ore set of homogeneous elements, which is graded isomorphic to the $\mathbb{K}$-quantum torus $\mathcal{T}$ with generators $z_{j}^{ \pm 1}$ (of weights $\beta_{i_{j}}$ ), $j \in D^{\prime}$ for some subset $D^{\prime} \subseteq[1, l]$ and relations $z_{j} z_{j^{\prime}}=$ $q^{-\left\langle\beta_{i_{j}}, \beta_{i_{j^{\prime}}}\right\rangle} z_{j^{\prime}} z_{j}, \forall j>j^{\prime} \in D^{\prime}$. Since $\mathcal{T}$ is obtained from $\mathcal{U}_{-}^{w} / I_{w}(y)$ by the localization by homogeneous elements, $u$ is a diagonal normal element of $\mathcal{T}$. Let $D^{\prime}=\left\{j_{1}<\ldots<j_{k}\right\}$. Write $u$ as a sum of monomials $t z_{j_{1}}^{m_{1}} \ldots z_{j_{k}}^{m_{k}}, t \in \mathbb{K}^{*}, m_{1}, \ldots, m_{k} \in \mathbb{Z}$. Let $t z_{j_{1}}^{m_{1}} \ldots z_{j_{k}}^{m_{k}}$ be one such monomial that occurs in $u$. By Theorem 4.3 the lattice generated by $\beta_{i j}, j \in D^{\prime}$ is equal to $\mathcal{Q}_{y, w}$. Since $u$ is a diagonal normal element,

$$
u z_{n}=q^{\left\langle m_{1} \beta_{i_{1}}+\ldots+m_{n-1} \beta_{i_{j_{n-1}}}-m_{n+1} \beta_{i_{j_{n+1}}}-\ldots-m_{k} \beta_{i_{j_{k}}}, \beta_{i_{j_{n}}}\right\rangle} z_{n} u, \quad \forall n \in[1, k],
$$

and

$$
\begin{aligned}
\beta_{i_{j_{n}}} & \in \mathcal{Q}_{y, w} \mapsto\left\langle m_{1} \beta_{i_{j_{1}}}+\ldots+m_{n-1} \beta_{i_{j_{n-1}}}-m_{n+1} \beta_{i_{j_{n+1}}}-\ldots-m_{k} \beta_{i_{j_{k}}}, \beta_{i_{j_{n}}}\right\rangle \in \mathbb{Z}, \\
n & \in[1, k]
\end{aligned}
$$

defines a group homomorphism. Therefore, there exists $\mu_{0} \in\left(1 / n_{y, w}\right) \mathcal{P}$ such that

$$
u x=q^{\left\langle\mu_{0}, \gamma\right\rangle} x u, \quad \forall x \in \mathcal{T}_{\gamma}, \gamma \in \mathcal{Q}_{y, w} .
$$

This implies that (4.8) holds for all $x \in\left(\mathcal{U}_{-}^{w} / I_{w}(y)\right)_{\gamma}$ and thus for all $x \in\left(R_{y, w}\right)_{\gamma}$. Let $u \in\left(\mathcal{U}_{-}^{w} / I_{w}(y)\right)_{\gamma_{0}}, \gamma_{0} \in \mathcal{Q}_{y, w}$. Using (4.2) we obtain that for all $\lambda \in \mathcal{P}$,

$$
u a_{y, w}^{\lambda}=q^{\left\langle\mu_{0},(w-y) \lambda\right\rangle} a_{y, w}^{\lambda} u=q^{\left\langle\mu_{0},(w-y) \lambda\right\rangle} q^{-\left\langle(w+y) \lambda, \gamma_{0}\right\rangle} u a_{y, w}^{\lambda} .
$$

Since $q \in \mathbb{K}^{*}$ is not a root of unity $\left\langle\mu_{0},(w-y) \lambda\right\rangle-\left\langle(w+y) \lambda, \gamma_{0}\right\rangle=0$, i.e.

$$
\left\langle\lambda, w^{-1}\left(\mu_{0}-\gamma_{0}\right)\right\rangle=\left\langle\lambda, y^{-1}\left(\mu_{0}+\gamma_{0}\right)\right\rangle, \quad \forall \lambda \in \mathcal{P} .
$$


Thus, $w^{-1}\left(\mu_{0}-\gamma_{0}\right)=y^{-1}\left(\mu_{0}+\gamma_{0}\right)$ and

$$
\left(w y^{-1}-1\right)\left(-\mu_{0}\right)=\left(w y^{-1}+1\right) \gamma_{0} .
$$

By the standard linear algebra for Cayley transforms there exits $\mu \in \operatorname{Span}_{\mathbb{Q}}\left\{\alpha_{i}\right\}_{i=1}^{r}$ such that

$$
\gamma_{0}=\left(w y^{-1}-1\right) y \mu=(w-y) \mu \text { and }-\mu_{0}=\left(w y^{-1}+1\right) y \mu=(w+y) \mu
$$

(see e.g. the proof of [40, Theorem 3.6]). We have $w \mu=\left(\gamma_{0}-\mu_{0}\right) / 2 \in\left(1 / 2 n_{y, w}\right) \mathcal{P}$, so that $\mu \in\left(1 / 2 n_{y, w}\right) \mathcal{P}$. Furthermore, $(w-y) \mu=\gamma_{0} \in \mathcal{Q}_{\mathcal{S}(w)}$ and $\left\langle(w+y) \mu, \mathcal{Q}_{y, w}\right\rangle=$ $\left\langle-\mu_{0}, \mathcal{Q}_{y, w}\right\rangle \subseteq \mathbb{Z}$, so $\mu$ satisfies all the required properties. This completes the proof of the proposition.

By developing further the arguments of the proof of Proposition 4.4 we will classify all normal elements of the localizations $R_{y, w}$ in a forthcoming publication.

4.4. The main result. Denote the lattice

$$
\mathcal{L}_{y, w, \mathbf{p}}=\left\{(w-y) \mu \mid \mu \in \mathcal{P}, \mathbf{r}((w-y) \mu, \gamma) q^{-\langle(w+y) \mu, \gamma\rangle}=1, \forall \gamma \in \mathcal{Q}_{y, w}\right\},
$$

recall (4.6). One can equivalently use only $\mu \in \mathcal{P}_{\mathcal{S}(w)}$ in (4.9) because of (2.21).

The following theorem provides an explicit formula for the dimensions of the Goodearl-Letzter strata $\operatorname{Spec}_{I_{w}(y)} \mathcal{U}_{-, \mathbf{p}}^{w}$ and completes the set theoretic description of the spectra of all multi-parameter quantum Schubert cell algebras $\mathcal{U}_{-, \mathbf{p}}^{w}$.

THEOREM 4.5. For all base fields $\mathbb{K}, q \in \mathbb{K}^{*}$ not a root of unity, Weyl group elements $y \leq w$ and 2-cocycles $\mathbf{p} \in Z^{2}\left(\mathcal{Q}_{\mathcal{S}(w)}, \mathbb{K}^{*}\right)_{n}$, the Goodearl-Letzter strata $\operatorname{Spec}_{I_{w}(y)} \mathcal{U}_{-, \mathbf{p}}^{w}$ are homeomorphic to the spectra of Laurent polynomial rings over $\mathbb{K}$ of dimension equal to the rank of the lattice $\mathcal{L}_{y, w, \mathbf{p}}^{u}$, see (4.9).

Proof. Recall the definition of $n_{y, w} \in \mathbb{Z}_{+}$from Section 5 and define the lattice

$$
\begin{gathered}
\mathcal{L}_{y, w, \mathbf{p}}^{\prime}=\left\{(w-y) \mu \mid \mu \in\left(1 / 2 n_{y, w}\right) \mathcal{P},(w-y) \mu \in \mathcal{Q}_{\mathcal{S}(w)},\left\langle(w+y) \mu, \mathcal{Q}_{y, w}\right\rangle \subseteq \mathbb{Z},\right. \\
\left.\mathbf{r}((w-y) \mu, \gamma) q^{-\langle(w+y) \mu, \gamma\rangle}=1, \forall \gamma \in \mathcal{Q}_{y, w}\right\} .
\end{gathered}
$$

It follows from (4.2) that $a_{y, w}^{\mu} \in\left(\mathcal{Z}_{y, w}\right)_{(w-y) \mu} \backslash\{0\}$ for all $\mu \in \mathcal{P}$ that satisfy the equation in (4.9). Therefore, $\mathcal{Z}_{y, w} \supseteq \mathcal{L}_{y, w, \mathbf{p}}$. Since every central element of a graded ring is a diagonal normal element, Proposition 4.4 implies that $\mathcal{Z}_{y, w} \subseteq \mathcal{L}_{y, w, \mathbf{p}}^{\prime}$. Therefore,

$$
\mathcal{L}_{y, w, \mathbf{p}} \subseteq \mathcal{Z}_{y, w} \subseteq \mathcal{L}_{y, w, \mathbf{p}}^{\prime}
$$

One easily shows that the index $\left[\mathcal{L}_{y, w, \mathbf{p}}^{\prime}: \mathcal{L}_{y, w, \mathbf{p}}\right]$ is finite, starting from $[\mathcal{P}$ : $\left.\left(1 / 2 n_{y, w}\right) \mathcal{P}\right]<\infty$. Therefore,

$$
\operatorname{rank} \mathcal{L}_{y, w, \mathbf{p}}=\operatorname{rank} \mathcal{Z}_{y, w}=\operatorname{rank} \mathcal{L}_{y, w, \mathbf{p}}^{\prime}
$$

The theorem follows from fact (4.4) that $Z\left(R_{y, w}\right)$ is a Laurent polynomial ring of dimension $\operatorname{rank} \mathcal{Z}_{y, w}$ and Theorem 3.1(d).

REMARK 4.6. Cauchon [7] proved that for a Cauchon-Goodearl-Letzter (CGL) extensions $R$ and a torus invariant prime ideal $I$ of $R, R / I$ admits a localization, which 
is isomorphic to a quantum torus. Computing the dimension of the corresponding Goodearl-Letzter stratum amounts to computing the dimension of its centre. Bell and Launois applied this to obtain a formula in [2, Proposition 3.3] for uniparameter CGL extensions, which correspond to cases when the Cauchon localization is a special kind of quantum torus with exchange relations involving powers of $q$. For cocycles $\mathbf{p}$ taking values in the cyclic subgroup of $\mathbb{K}^{*}$ generated by $q$, this leads to a formula for the Goodearl-Letzter strata of $\mathcal{U}_{-, \mathbf{p}}^{w}$ in terms of the dimensions of the kernels of large square matrices of size $\ell(w)-\ell(y)$, which is less efficient than Theorem 4.5.

\section{Equivariant polynormality, normal separation and catenarity of $\mathcal{U}_{-, \mathrm{p}}^{w}$.}

5.1. Polynormality. In this section, using results of our previous paper [41], we prove that all $\mathbb{T}^{|\mathcal{S}(w)|}$-prime ideals of $\mathcal{U}_{-, \mathbf{p}}^{w}$ are equivariantly polynormal, the spectra Spec $\mathcal{U}_{-, \mathbf{p}}^{w}$ are normally separated and all algebras $\mathcal{U}_{-, \mathbf{p}}^{w}$ are catenary.

We start with a brief review of equivariant polynormality. Assume that a ring $R$ is equipped with an action of a group $\Gamma$ by algebra automorphism. We say that an element $u \in R$ is $\Gamma$-normal if it is a $\Gamma$-eigenvector and if there exists $g \in \Gamma$ such that $u r=(g . r) u$ for all $r \in R$. Sometimes $\Gamma$-normality is defined requiring only the second condition, see [13], but this is not sufficient to extend the definition to $\Gamma$-polynormality for $\Gamma$ stable ideals of $R$. Given a $\Gamma$-stable ideal $I$ of $R$, we say that a sequence $u_{1}, \ldots, u_{N} \in R$ is a $\Gamma$-polynormal generating sequence of $I$ if $\left\{u_{1}, \ldots, u_{N}\right\}$ generates $I$ and for all $i=1, \ldots, N$ the element $u_{i}$ is a $\Gamma$-normal element of $R$ modulo the ideal generated by $u_{1}, \ldots, u_{i-1}$. Note that it follows from the conditions on the elements $u_{1}, \ldots, u_{i-1}$ that the two-sided ideal of $R$ generated by them is $\Gamma$-stable and equals $R u_{1}+\ldots+R u_{i}$. In particular, $R$ is generated by $u_{1}, \ldots, u_{N}$ as a one-sided (left or right) ideal.

For all $\mu \in \mathcal{P}_{\mathcal{S}(w)}, \vartheta \in \mathcal{Q}_{\mathcal{S}(w)}$ there exists a unique $t_{\mu, \vartheta} \in \mathbb{T}^{|\mathcal{S}(w)|}$ such that

$$
\left(t_{\mu, \vartheta}\right)^{\gamma}=\mathbf{r}(\vartheta, \gamma) q^{\langle\vartheta-2 w \mu, \gamma\rangle}, \quad \forall \gamma \in \mathcal{Q}_{\mathcal{S}(w)},
$$

cf. (2.22) and (2.26). It is clear that the map $(\mu, \vartheta) \in \mathcal{P}_{\mathcal{S}(w)} \times \mathcal{Q}_{\mathcal{S}(w)} \mapsto t_{\mu, \vartheta} \in \mathbb{T}^{|\mathcal{S}(w)|}$ is a group homomorphism. Thus, its image

$$
\mathcal{P} \mathcal{Q}_{w, \mathbf{p}}=\left\{t_{\mu, \vartheta} \mid \mu \in \mathcal{P}_{\mathcal{S}(w)}, \vartheta \in \mathcal{Q}_{\mathcal{S}(w)}\right\}
$$

is a subgroup of $\mathbb{T}^{|\mathcal{S}(w)|}$, which is a quotient of $\mathcal{P}_{\mathcal{S}(w)} \times \mathcal{Q}_{\mathcal{S}(w)}$.

For every $\lambda \in \mathcal{P}_{\mathcal{S}(w)}^{+}$and $y \leq w \in W$ fix a finite subset $\mathcal{V}_{y, w}(\lambda)^{*} \subset V(\lambda)^{*}$ consisting of homogeneous elements such that the restriction map

$$
\left.\xi \in V(\lambda)^{*} \mapsto \xi\right|_{\mathcal{U}_{+} T_{w} v_{\lambda}} \in\left(\mathcal{U}_{+} T_{w} v_{\lambda}\right)^{*}
$$

sends bijectively $\mathcal{V}_{y, w}(\lambda)^{*}$ to a basis of $\left(\mathcal{U}_{-} T_{y} v_{\lambda} \cap \mathcal{U}_{+} T_{w} v_{\lambda}\right)^{\perp} \subset\left(\mathcal{U}_{+} T_{w} v_{\lambda}\right)^{*}$. Given $\Omega \subset$ $\mathcal{P}_{\mathcal{S}(w)}^{+}$denote

$$
\mathcal{V}_{y, w}(\Omega)=\bigsqcup_{\lambda \in \Omega} \mathcal{V}_{y, w}(\lambda)
$$

Define the maps

$$
\mathrm{hw}: \mathcal{V}_{y, w}(\Omega) \rightarrow \Omega, \mathrm{wt}: \mathcal{V}_{y, w}(\omega) \rightarrow \mathcal{P}, \quad \operatorname{hw}(\xi)=\lambda, \operatorname{wt}(\xi)=\nu, \quad \text { if } \xi \in\left(V(\lambda)^{*}\right)_{\nu}
$$


Denote the partial ordering on $\mathcal{V}_{y, w}(\Omega)$ :

$$
\xi \preceq \xi^{\prime}, \text { if } \operatorname{hw}(\xi)=\operatorname{hw}\left(\xi^{\prime}\right) \text { and } \operatorname{wt}(\xi) \leq \operatorname{wt}\left(\xi^{\prime}\right)
$$

THEOREM 5.1. Assume that $\mathbb{K}$ is an arbitrary base field, $q \in \mathbb{K}^{*}$ is not a root of unity, $\mathfrak{g}$ is an arbitrary simple Lie algebra, $w \in W$ and $\mathbf{p} \in Z^{2}\left(\mathcal{Q}_{\mathcal{S}(w)}, \mathbb{K}^{*}\right)_{n}$ is a normalized 2-cocycle.

(a) Since $\mathcal{U}_{-, \mathbf{p}}^{w}$ is noetherian, for each $y \in W^{\leq w}$ there exists a finite subset $\Omega$ of $\mathcal{P}_{\mathcal{S}(w)}^{+}$ such that the ideal $I_{w}(y)$ is generated by $b_{w, \xi}^{\lambda}$ for $\lambda \in \Omega, \xi \in V(\lambda)^{*}$, recall (3.6). Fix any linear ordering $\xi_{1}<\ldots<\xi_{N}$ on $\mathcal{V}_{y, w}(\Omega)$, which is a refinement of partial ordering (5.2). Then

$$
b_{w, \xi_{1}}^{\mathrm{hw}\left(\xi_{1}\right)}, \ldots, b_{w, \xi_{N}}^{\mathrm{hw}\left(\xi_{N}\right)}
$$

is $a \mathbb{T}^{|\mathcal{S}(w)|}$-polynormal generating sequence of the ideal $I_{w}(y)$. More precisely,

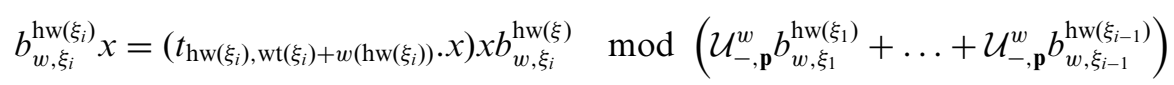

for all $x \in \mathcal{U}_{-, \mathrm{p}}^{w}, i=1, \ldots, N$. All automorphism related to the above normal elements come from the subgroup $\mathcal{P} \mathcal{Q}_{w, \mathbf{p}}$ of $\mathbb{T}^{|\mathcal{S}(w)|}$, cf. (5.1).

(b) If the base field $\mathbb{R}$ has characteristic 0 and $q$ is transcendental over $\mathbb{Q}$, then the conclusion of part (a) is valid for $\Omega=\left\{\omega_{i} \mid i \in \mathcal{S}(w)\right\}$.

Proof. The untwisted cases of parts (a) and (b) of Theorem 5.1 were proved in [41, Theorem 3.6] and [41, Theorem 3.4], respectively. The theorem follows from this, since all elements of the polynormal generating sets are homogeneous with respect to the grading (2.3).

A special case of Theorem 5.1 gives a constructive proof of a conjecture of Brown and Goodearl, [4, Conjecture II.10.9], that the torus invariant prime ideals of the multiparameter algebras of quantum matrices have polynormal generating sets consisting of quantum minors. (This is an extension of the Goodearl-Lenagan conjecture [16] on polynormality in single parameter quantum matrix algebras, which we proved in [41].) Artin et al. showed [1, p. 889] (see also [15, Lemma 3.6]) that the multi-parameter algebras of quantum matrices $\mathcal{O}_{\lambda, \mathbf{p}}\left(M_{m, n}(\mathbb{K})\right)$ are obtained by twists from the single parameter ones if $\lambda$ is a square root of $q$ in $\mathbb{K}$ (provided that such exists). In [33, Proposition 2.1.1] (and [41, Lemma 4.1]) the single parameter algebras of quantum matrices were realized as special cases of the algebras $\mathcal{U}_{+}^{w}\left(\right.$ and $\left.\mathcal{U}_{-}^{w}\right)$ in a way that matches the corresponding gradings by free abelian groups. Therefore, the isomorphism of $[\mathbf{4 1}$, Lemma 4.1] realizes $\mathcal{O}_{\lambda, \mathbf{p}}\left(M_{m, n}(\mathbb{K})\right)$ as a special case of the algebras $\mathcal{U}_{-, \mathbf{p}}^{w}$. In this case by [41, Lemma 4.3] if char $\mathbb{K}=0$ and $q$ is transcendental over $\mathbb{Q}$, the generating sets from Theorem 5.1(b) consist of quantum minors, which are explicitly listed in [41, Theorem 4.4]. More generally, for all simple Lie algebras $\mathfrak{g}$ and Weyl group elements $w$, if char $\mathbb{K}=0$ and $q$ is transcendental over $\mathbb{Q}$, then in Theorem 5.1 one can choose $\Omega=\left\{\omega_{1}, \ldots, \omega_{r}\right\}$ by [41, Theoerm 3.4]. The last two facts rely on the theorem of Joseph [28, Théorème 3] and need the stronger assumption on $\mathbb{K}$ and $q$ because of a specialization argument. 
5.2. Catenarity. Finally we establish normal separation of $\operatorname{Spec} \mathcal{U}_{-, \mathbf{p}}^{w}$ and catenarity of $\mathcal{U}_{-, \mathbf{p}}^{w}$.

THEOREM 5.2. For all base fields $\mathbb{K}, q \in \mathbb{K}^{*}$ not a root of unity, simple Lie algebras $\mathfrak{g}$, $w \in W$ and 2-cocycles $\mathbf{p} \in Z^{2}\left(\mathcal{Q}_{\mathcal{S}(w)}, \mathbb{K}^{*}\right)_{n}$, the spectra of the algebras $\mathcal{U}_{-, \mathbf{p}}^{w}$ are normally separated.

Proof. Using Goodearl's result [13, Corollary 4.6] it suffices to show that $\mathcal{U}_{-, \mathbf{p}}^{w}$ has graded normal separation. This can be proved in two different ways. Firstly, assume that $I_{1} \subsetneq I_{2}$ are two graded (i.e. $\mathbb{T}^{|\mathcal{S}(w)|}$-invariant) prime ideals of $\mathcal{U}_{-, \mathbf{p}}^{w}$. If $x_{1}, \ldots, x_{n}$ is a $\mathbb{T}^{|\mathcal{S}(w)|}$-polynormal generating sequence of $I_{2}$ as in Theorem 5.1(a), then the first $x_{i}$, which does not belong to $I_{1}$, produces a graded normal separating element for the pair of ideals $I_{1}, I_{2}$. Secondly, by Theorem 3.1(a) for every pair $I_{1} \subsetneq I_{2}$ of graded prime ideals of $\mathcal{U}_{-, \mathbf{p}}^{w}$, there exist $y_{1}<y_{2} \in W^{\leq w}$ such that $I_{1}=I_{w}\left(y_{1}\right)$ and $I_{2}=I_{w}\left(y_{2}\right)$. Let $\lambda \in \mathcal{P}^{++}$. Then $y_{1} \lambda>y_{2} \lambda$, so $a_{y_{1}, w}^{\lambda} \in I_{w}\left(y_{2}\right)$. Theorem 3.1(c) implies that $a_{y_{1}, w}^{\lambda} \notin I_{w}\left(y_{1}\right)$, and equation (3.1) gives that $a_{y_{1}, w}^{\lambda}$ is normal modulo $I_{w}\left(y_{1}\right)$.

Recall that a ring $R$ is Auslander-Gorenstein if the injective dimension of $R$ (as both right and left $R$-module) is finite, and for all integers $0 \leq i<j$ and finitely generated (right or left) $R$-modules $M$, we have $\operatorname{Ext}_{R}^{i}(N, R)=0$ for all $R$-submodules $N$ of $\operatorname{Ext}_{R}^{j}(M, R)$. A ring $R$ is Auslander regular if, in addition, the global dimension of $R$ is finite. The grade of a finitely generated $R$-module $M$ is given by

$$
j(M)=\inf \left\{i \geq 0 \mid \operatorname{Ext}_{R}^{i}(M, R) \neq 0\right\} .
$$

An algebra $R$ is Cohen-Macauley if

$$
j(M)+\mathrm{GK} \operatorname{dim} M=\mathrm{GK} \operatorname{dim} R
$$

for all finitely generated $R$-modules $M$. We will need the following two results.

TheOREm 5.3. ([14]). Assume that A is an affine, noetherian, Auslander-Gorenstein and Cohen-Macaulay algebra over a field with finite Gelfand-Kirillov dimension. If $\operatorname{Spec} A$ is normally separated, then $A$ is catenary. If, in addition, $A$ is a prime ring, then Tauvel's height formula holds.

Tauvel's height formula holds for a ring $R$ if for all prime ideals $J$ of $R$, the height of $J$ is equal to

$$
\mathrm{GK} \operatorname{dim} R-\mathrm{GK} \operatorname{dim}(R / J) .
$$

THEOREM 5.4. ([11, 29]). Assume $R$ is a noetherian, Auslander regular ring. Let $S=R[x ; \sigma, \delta]$ be an Ore extension of $R$. Then:

(a) $S$ is Auslander regular.

(b) If $R=\oplus_{k \geq 0} R_{k}$ is a connected graded Cohen-Macauley $\mathbb{K}$-algebra over a field $\mathbb{K}$ such that $\sigma\left(R_{k}\right) \subseteq R_{k}$ for all $k \geq 0$, then $S$ is Cohen-Macauley.

The next theorem proves that all algebras $\mathcal{U}_{-, \mathbf{p}}^{w}$ are catenary.

THEOREM 5.5. For all base fields $\mathbb{K}, q \in \mathbb{K}^{*}$ not a root of unity, simple Lie algebras $\mathfrak{g}$, $w \in W$ and and 2-cocycles $\mathbf{p} \in Z^{2}\left(\mathcal{Q}_{\mathcal{S}(w)}, \mathbb{K}^{*}\right)_{n}$, the algebras $\mathcal{U}_{-, \mathbf{p}}^{w}$ are Auslander regular, Cohen-Macauley, catenary, and Tauvel's height formula holds for them. For all $y \in W \leq w$ 
the height of prime ideal $I_{w}(y)$ is equal to $\ell(y)$ and

$$
\operatorname{GK} \operatorname{dim}\left(\mathcal{U}_{-, \mathbf{p}}^{w} / I_{w}(y)\right)=\ell(w)-\ell(y) .
$$

Proof. Lemma 2.4(a)-(b) and Theorem 5.4(a) imply that $\mathcal{U}_{-, \mathbf{p}}^{w}$ is Auslander regular. Given $\lambda \in \mathcal{P}^{++}$, we can specialize the $-\mathcal{Q}_{\mathcal{S}(w)}^{+}$-grading of $\mathcal{U}_{-, \mathbf{p}}^{w}$ from (2.3) to an $\mathbb{N}$ grading by

$$
\left(\mathcal{U}_{-, \mathbf{p}}^{w}\right)_{n}=\left\{x \in\left(\mathcal{U}_{-, \mathbf{p}}^{w}\right)_{-\gamma} \mid \gamma \in \mathcal{Q}_{\mathcal{S}(w)}^{+},\langle\lambda, \gamma\rangle=n\right\}, \quad n \in \mathbb{N} .
$$

Obviously, the $\mathbb{T}^{|\mathcal{S}(w)|}$-action (2.23) preserves each graded component $\left(\mathcal{U}_{-, \mathbf{p}}^{w}\right)_{n}$ and $\mathcal{U}_{-, \mathbf{p}}^{w}$ is connected. Lemma 2.4(a)-(b) and Theorem 5.4(b) imply that $\mathcal{U}_{-, \mathbf{p}}^{w}$ is Cohen-Macauley. It also follows from Lemma 2.4(a)-(b) that the algebras $\mathcal{U}_{-, \text {p }}^{w}$ are affine, noetherian domains with $\mathrm{GK} \operatorname{dim} \mathcal{U}_{-, \mathbf{p}}^{w}=\ell(w)$. Theorem 5.3 implies that all algebras $\mathcal{U}_{-, \mathbf{p}}^{w}$ are catenary and satisfy Tauvel's height formula.

Since the Gelfand-Kirillov dimension of a graded algebra does not change under twisting, the last statement of the theorem follows from [41, Theorem 5.8].

Some special cases of the untwisted case of Theorems 5.1, 5.2 and 5.5 were established by Caldero [6], Cauchon [8], Goodearl-Lenagan [14] and Malliavin [31]. The general untwisted case of Theorems 5.1, 5.2 and 5.5 was proved by Yakimov in [41].

ACKNOwedgement. I am thankful to the organizers of the conference "New developments in noncommutative algebra and its applications" Skye, Scotland for the invitation to participate, and to the referee whose comments helped me to improve the exposition of the paper. The author was supported in part by NSF grant DMS1001632 .

\section{REFERENCES}

1. M. Artin, W. Schelter and J. Tate, Quantum deformations of $\mathrm{GL}_{n}$, Comm. Pure Appl. Math. 44 (1991), 879-895.

2. J. P. Bell and S. Launois, On the dimension of $H$-strata in quantum algebras, Algebra Number Theory 4 (2010), 175-200.

3. J. Bell, K. Casteels and S. Launois, Primitive ideals in quantum Schubert cells: dimension of the strata, Forum Math. (in print) doi:10.1515/forum-2011-0155.

4. K. A. Brown and K. R. Goodearl, Lectures on algebraic quantum groups, Advanced Courses in Mathematics (CRM. Barcelona, Spain, 2002).

5. K. A. Brown, K. R. Goodearl and M. Yakimov, Poisson structures of affine spaces and flag varieties. I. Matrix affine Poisson space, Adv. Math. 206 (2006), 567-629. 444-457.

6. P. Caldero, Étude des $q$-commutations dans l'algèbre $U_{q}\left(\mathfrak{n}^{+}\right)$, J. Algebra 178 (1995),

7. G. Cauchon, Effacement des dérivations et spectres premiers d'algébres quantiques, J. Algebra 260 (2003), 476-518.

8. G. Cauchon, Spectre premier de $O_{q}\left(M_{n}(k)\right)$ : image canonique et séparation normale, J. Algebra 260 (2003), 519-569.

9. C. De Concini, V. Kac and C. Procesi, Some quantum analogues of solvable Lie groups, in Geometry and analysis (Tata Inst. Fund. Res., Bombay, India, 1992), pp. 41-65.

10. M. Costantini and M. Varagnolo, Quantum double and multiparameter quantum groups, Comm. Algebra 22 (1994), 6305-6321. 
11. E. K. Ekström, The Auslander condition on graded and filtered Noetherian rings, in Séminaire d'Algèbre Paul Dubreil et Marie-Paul Malliavin, Lecture Notes in Math., 1404 (Springer, Berlin, Germany, 1989), 220-245.

12. C. Geiß, B. Leclerc and J. Schröer, Cluster structures on quantized coordinate rings, Selecta Math. 19 (2013), 337-397.

13. K. R. Goodearl, Prime spectra of quantized coordinate rings, in Interactions between ring theory and representations of algebras (Murcia), Lect. Notes in Pure and Appl. Math., 210 (Dekker, New York, 2000), 205-237.

14. K. R. Goodearl and T. H. Lenagan, Catenarity in quantum algebras, J. Pure Appl. Algebra 111 (1996), 123-142.

15. K. R. Goodearl and T. H. Lenagan, Quantum determinantal ideals, Duke Math. J. 103 (2000), 165-190.

16. K. R. Goodearl and T. H. Lenagan, Winding-invariant prime ideals in quantum $3 \times 3$ matrices, J. Algebra 260 (2003), 657-687.

17. K. R. Goodearl and E. S. Letzter, Prime factor algebras of the coordinate ring of quantum matrices, Proc. Amer. Math. Soc. 121 (1994), 1017-1025.

18. K. R. Goodearl and E. S. Letzter, The Dixmier-Moeglin equivalence in quantum coordinate rings and quantized Weyl algebras, Trans. Amer. Math. Soc. 352 (2000) 1381-1403.

19. K. R. Goodearl and M. Yakimov, Poisson structures of affine spaces and flag varieties. II, Trans. Amer. Math. Soc. 361 (2009), 5753-5780.

20. M. Gorelik, The prime and the primitive spectra of a quantum Bruhat cell translate, J. Algebra 227 (2000), 211-253.

21. T. J. Hodges, T. Levasseur and M. Toro, Algebraic structure of multiparameter quantum groups, Adv. Math. 126 (1997), 52-92.

22. I. Heckenberger and H.-J. Schneider, Right coideal subalgebras of Nichols algebras and the Duflo order on the Weyl groupoid, Israel J. Math. (in print) doi:10.1007/s11856-012-0180-3.

23. K. L. Horton, The prime and primitive spectra of multiparameter quantum symplectic and Euclidean spaces, Comm. Algebra 31 (2003), 4713-4743.

24. J. C. Jantzen, Lectures on quantum groups, Grad. Studies in Math., 6 (American Mathematical Society, Providence, RI, 1996).

25. G. Johnson and C. Nowlin, The FRT-construction via quantum affine algebras and smash products, J. Algebra 353 (2012), 158-173.

26. A. Joseph, On the prime and primitive spectra of the algebra of functions on a quantum group, J. Algebra 169 (1994), 441-511.

27. A. Joseph, Quantum groups and their primitive ideals, Ergebnisse der Mathematik und ihrer Grenzgebiete 3 (Springer-Verlag, Berlin, Germany, 1995).

28. A. Joseph, Sur les idéaux génériques de l'agèbre des fonctions sur un groupe quantique, C. R. Acad. Sci. Paris Sér. I. Math. 321 (1995), 135-140.

29. T. Levasseur and J. T. Stafford, The quantum coordinate ring of the special linear group, J. Pure Appl. Algebra 86 (1993), 181-186.

30. G. Lusztig, Introduction to quantum groups, Progress in Mathematics, 110 (Birkhäuser, Boston, MA, 1993).

31. M.-P. Malliavin, La caténarité de la partie positive de l'algèbre enveloppante quantifiée de l'algèbre de Lie simple de type $B_{2}$, Beiträge Algebra Geom. 35 (1994), 73-83 (Festschrift on the occasion of the 65th birthday of Otto Krötenheerdt).

32. R. J. Marsh and K. Rietsch, Parametrizations of flag varieties, Represent. Theory 8 (2004), 212-242.

33. A. Mériaux and G. Cauchon, Admissible diagrams in $U_{q}^{w}(\mathfrak{g})$ and combinatoric properties of Weyl groups, Represent. Theory 14 (2010), 645-687.

34. C. Nowlin, Torus-invariant prime spectra of affine quantum nilpotent algebras, $\mathrm{PhD}$ thesis(University of California, Santa Barbara, CA, 2010).

35. S.-Q. Oh, Quantum and Poisson structures of multi-parameter symplectic and Euclidean spaces, J. Algebra 319 (2008), 4485-4535.

36. X. Tang, The prime ideal stratification and the automorphism group of $U_{r, s}^{+}\left(B_{2}\right)$ (preprint) arXiv:1109.2640.

37. M. Yakimov, A classification of $H$-primes of quantum partial flag varieties, Proc. Amer. Math. Soc. 138 (2010), 1249-1261. 
38. M. Yakimov, Invariant prime ideals in quantizations of nilpotent Lie algebras, Proc. London Math. Soc. 101(2) (2010), 454-476.

39. M. Yakimov, Strata of prime ideals of De Concini-Kac-Procesi algebras and Poisson geometry, in New trends in noncommutative algebra, Contem. Math. 562 (P. Ara, K. A. Brown, T. H. Lenagan, E. S. Letzter, J. T. Stafford and J. J. Zhang, Editors) (American Mathematical Society, Providence, RI, 2012), 265-278.

40. M. Yakimov, On the spectra of quantum groups, Mem. Amer. Math. Soc. (in print), arXiv: 1106.3821.

41. M. Yakimov, A proof of the Goodearl-Lenagan polynormality conjecture, Int. Math. Res. Not. 2013(9) (2013), 2097-2132. 